\title{
Biodiversity and seasonal variation of benthic macrofauna in Minicoy Island, Lakshadweep, India
}

\author{
Dalia Susan $V^{1}$, Satheesh Kumar P. ${ }^{*}$, Pillai N. G. K ${ }^{1}$ \\ ${ }^{1}$ Central Marine Fisheries Research Institute, Kochi, Kerala 682018, India \\ Received 13 September 2013; accepted 14 April 2014
}

(CThe Chinese Society of Oceanography and Springer-Verlag Berlin Heidelberg 2014

\begin{abstract}
From the Indian coast only limited data are available on the benthic fauna of the seagrass communities. In this study, seasonal variation in the distribution of macrobenthos and influence of environmental parameters was explored at four seagrass beds and two mangrove stations along the Minicoy Island, Lakshadweep, India, from September 1999 to August 2001. A total of 160 macrobenthic species from eight major groups represented the macrofauna of the Minicoy Island. Of the identified taxa, molluscs 70 (gastropods 41.46\%, bivalves $7.5 \%)$, polychaetes $27(16.88 \%)$, crustaceans $30(18.75 \%)$, echinoderms $11(6.88 \%)$ and remaining others. Average seasonal abundance of benthic macrofauna ranged from 219 to $711 \mathrm{ind} . / \mathrm{m}^{2}$, species diversity varied from 1.45 to 3.64 bits per individual, species richness index ranged from 4.01 to 26.17 , evenness 0.69 to 1.66 . In general, the higher abundance and species diversity was noticed in southern seagrass stations and northern seagrass stations, but in the mangrove stations comparatively low species diversity was observed. Three-way analysis of variance indicated that all communities resulted as being significantly different between seagrass and mangrove station, mainly when the seasonal interaction was considered. Multivariate analyses were employed to help define benthic characteristic and the relationship between environmental parameters at the six monitoring stations. Results of cluster analyses and multidimensional scale plot suggest that for mangrove region, different physiographic provinces, lower salinity, dissolved oxygen and sediment biotic structure have a higher influence on the species composition and diversity than other oceanographic conditions.
\end{abstract}

Key words: abundance, seagrass, macrobenthos, mangrove, Minicoy Island, Lakshadweep

Citation: Dalia Susan V, Satheesh Kumar P., Pillai N. G. K. 2014. Biodiversity and seasonal variation of benthic macrofauna in Minicoy Island, Lakshadweep, India. Acta Oceanologica Sinica, 33(10): 58-73, doi: 10.1007/s13131-014-0541-3

\section{Introduction}

The Lakshadweep group of Islands is located off the southwestern coast of India in the Arabian Sea. There are 36 Islands covering an area of $32 \mathrm{~km}^{2}$. From the Indian coast only limited information is available on the benthic fauna of the seagrass communities. The present study deals at Minicoy Island with the diversity and seasonal variations of macrobenthic fauna in seagrass and mangrove communities. The community studies on macro invertebrate abundance in Minicoy Island were limited to benthic macro and meiofauna of seagrass bed by Ansari 1984 during the cruise No. 104 of R/V Gaveshni. The study was based on one time collection up to the level of major benthic groups and no information is available on the benthic final association of this region. Ansari et al. (1991) conducted studies on the seagrass habitat complexity and abundance of macro invertebrate abundance in Lakshadweep coral regions. However, no work has been done to elucidate the mangrove benthic community structure of Minicoy Island. Mangrove region of Minicoy is limited to two patches of about $10^{4} \mathrm{~m}^{2}$ each. The mangroves noticed on Minicoy Island of Lakshadweep are in the formative stage and free from serious human interference.

Seagrass is marine flowering plants that occur in the near shore area around the world (Hemminga and Durate, 2000). Seagrass plays a key ecological role in the marine environment including organic carbon production and tropic transfers to adjacent habitats (Costanza et al., 1997). They are major pri- mary producers supporting the high productivity of associated animals including commercially important fish and large invertebrates as well as endangered marine animals sea turtles, dugongs and manatees (Heck et al., 2003). Seagrass also supports a variety of macrobenthic organisms such as polychaetes, mollusc and small crustaceans that play important roles in the food web, the decomposition process of beds and nutrient cycling (Williams and Heck, 2001).

In India the total area covered by mangroves is estimated at $6810 \mathrm{~km}^{2}$, which includes the adjacent mudflats and brackish water systems. Mangroves that exist in tropical and subtropical intertidal regions of the world support rich faunal resources and play an important role in the estuarine and coastal food webs (Alongi, 1990). Studies on the ecology of mangrove fauna of eastern and western coasts of India had been carried out (Khan et al., 2005; Saravanakumar et al., 2007; Kumar and Khan, 2013). Macrobenthos in marine sediment play an important role in ecosystem processes such as nutrient cycling, pollutant metabolism, dispersion and burial and in secondary production (Snelgrove, 1998). In the present study, an attempt has been made to understand the distribution and community structure of macrobenthos of seagrass beds and mangrove region. Environmental parameters such as temperature, salinity, dissolved oxygen and nutrients and grain size were also studied and correlated with the distribution and abundance of macrofauna. The benthic ecological data are required from unspoilt 
mangrove areas such as those on Minicoy Island as a baseline for meaningful and effective conservation measures.

\section{Materials and methods}

\subsection{Study site}

The Minicoy Island, located at $08^{\circ} 18^{\prime} \mathrm{N}$ and $73^{\circ} 04^{\prime} \mathrm{E}$ is the southernmost Island in the Lakshadweep group with a land area of $4.4 \mathrm{~km}^{2}$ and a length of $9.5 \mathrm{~km}$, tidal amplitude is approximately $1.75 \mathrm{~m}$. The present study concerns three sets of stations, southern seagrass stations (Thalassia bed, ThalassiaHalophila mixed bed), northern seagrass stations (Cymodaceae bed, Syringodium bed) and mangrove zone. Mangrove associated flora involves Avicinnia marina, Cereops tagal, Pemphis acidula and Bruguiera spp. Of the selected six stations, four were located in the intertidal zone (southern sea grass stations 1 and 2; northern seagrass stations 3 and 4 ) and two in the mangrove intertidal swamp (Fig. 1). Monthly triplicate samplings were collected during low tide from the above stations from September 1999 to August 2001. The details of GPS coordinates, vegetation, and soil substratum are presented in Table 1.

(1) Station 1, southern Thalassia bed located on the southern side of the Island and mainly constituted by a luxuriant growth of sea grass Thalassia spp.

(2) Station 2, southern Thalassia-Halophila bed was located on the south side about $100 \mathrm{~m}$ away from the high tide mark to the lagoon side.

(3) Station 3, northern Cymodaceae bed was located on the northwest side of the Island and had only a sparse growth of seagrass (Cymodaceae sp.). This was in the nearshore area partly protected from heavy wave action and currents by the 80-100 $\mathrm{m}$ wide zone of large coral conglomerates.

(4) Station 4, northern Syringodium bed was located on the northwest side of the Island almost $200 \mathrm{~m}$ away from the high tide mark in the lagoon and the vegetation was mainly constituted by Syringodium sp. of seagrass. This area was also partly protected by large coral conglomerates.

(5) Station 5, mangrove site bordered by Cereops tagal was located on the southwestern side of the Island near the helipad.

(6) Station 6, mangrove site bordered by Avicennia marina was located on the southwestern side of the Island, slightly away from the band and the banks were bordered by A. marina.

\subsection{Sampling methods}

Water samples were collected from the surface using a plastic bucket every month during low tide from all the stations for the measurement of temperature, salinity, $\mathrm{pH}$, dissolved oxygen and nutrients. Atmospheric temperature and seawater temperature were measured using a $0-50^{\circ} \mathrm{C}$ high precision thermometer. Salinity was determined by the Mohr's titrimetric method; silicate and nitrite present in seawater was estimated by Strickland and Parsons (1968). Dissolved oxygen was esti-

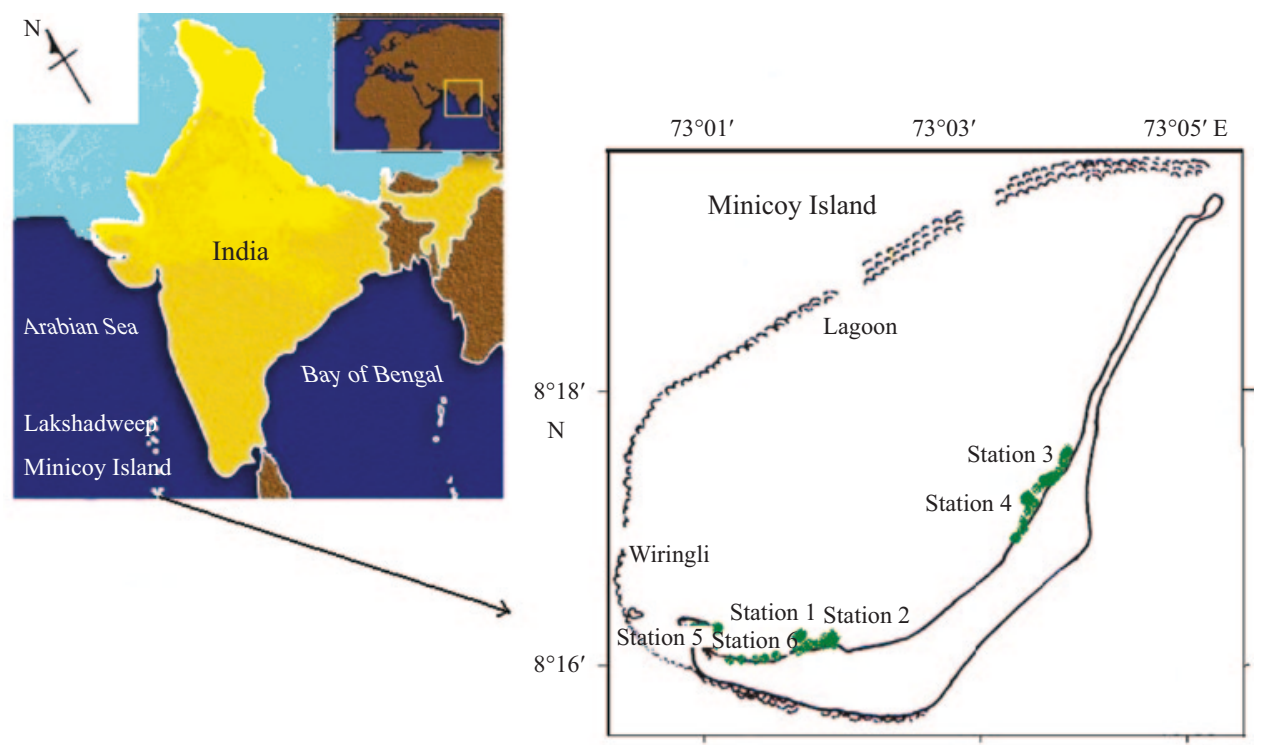

Fig.1. Location map of Minicoy Island showing the sampling stations.

Table 1. Details on GPS coordinates, seagrass and mangrove zone and soil substratum

\begin{tabular}{|c|c|c|c|c|}
\hline Study sites & North latitude & East longitude & Vegetation & Substratum \\
\hline Station 1 & $08^{\circ} 16^{\prime} 18^{\prime \prime}$ & $73^{\circ} 02^{\prime} 22^{\prime \prime}$ & southern Thalassia bed & sandy and silt \\
\hline Station 2 & $08^{\circ} 16^{\prime} 13^{\prime \prime}$ & $73^{\circ} 02^{\prime} 16^{\prime \prime}$ & southern Thalassia bed-Halophila bed & sand \\
\hline Station 3 & $08^{\circ} 16^{\prime} 32^{\prime \prime}$ & $73^{\circ} 02^{\prime} 45^{\prime \prime}$ & northern Cymodaceae bed & sand \\
\hline Station 4 & $08^{\circ} 16^{\prime} 35^{\prime \prime}$ & $73^{\circ} 02^{\prime} 41^{\prime \prime}$ & northern Syringodium bed & sandy and silt \\
\hline Station 5 & $08^{\circ} 16^{\prime} 09^{\prime \prime}$ & $73^{\circ} 02^{\prime} 12^{\prime \prime}$ & mangrove site bordered by Cereops tagal & silt and clay \\
\hline Station 6 & $08^{\circ} 16^{\prime} 10^{\prime \prime}$ & $73^{\circ} 02^{\prime} 14^{\prime \prime}$ & mangrove site bordered by Avicennia marina & silt and clay \\
\hline
\end{tabular}


mated employing modified Winkler method of Strickland and Parsons (1972). pH was measured using a pH meter (Mettler Toledo MP-120). Nitrate nitrogen in seawater was estimated by the method of Mullin and Riley (1955). Phosphorus present in seawater in the form of dissolved orthophosphate was determined quantitatively by the ascorbic acid method given by Murphy and Riley (1962). Each sediment sample brought to the laboratory was transferred to a glass dish and dried in an oven at $60^{\circ} \mathrm{C}$ and stored in desiccators for further analysis. Mechanical analysis by international pipette method was followed for grain size analysis (Krumbein and Pettijohn, 1983). Organic carbon was determined by the wet oxidation method of El-Wakeel and Riley (1957). Rainfall data of Minicoy were collected from the data sheets of Indian Meteorological Department.

\subsection{Benthic sampling and analytical methods}

Triplicate samples were collected every month using a metal quadrat of $25 \mathrm{~cm} \times 25 \mathrm{~cm}$ size up to a depth of $15 \mathrm{~cm}$ (Eleftheriou and Holme, 1984). The benthos in the sediment sample recovered after sieving through $0.5 \mathrm{~mm}$ mesh sieve was brought to the laboratory in polythene bags, transferred to a large white bottomed tray and the animals which were moving or easily recognizable were hand sorted. After this preliminary examination, the whole sample was treated with $5 \%$ buffered formalin and kept for further analysis. Fauna were identified to the lowest practical taxonomic level using standard references (Polychaeta: Day, 1967; Crabs: Williams, 1984; Mollusc: Fernando and Olivia Fernando, 2002). The numerical abundance was extrapolated into ind. $/ \mathrm{m}^{2}$ for easier data comparison. For the sake of interpreting the data, a calendar year was divided into three main seasons, pre monsoon (February-May), monsoon (June-September), and post monsoon (October-January). Biodiversity indices such as species diversity, richness and evenness were calculated following the standard formulae (Shannon and Weaver, 1963; Gleason, 1992; Pielou, 1966).

\subsection{Statistical analysis}

Three-way analysis of variance (ANOVA) was applied to the transformed data for testing the significance of differences and comparison among species, station and months (Snedecor and Cochran, 1967). Correlation between the macro benthic assemblage and environmental parameters was evaluated by Spearman correlation analysis. The significance of the models was tested using correlation coefficient. When the probability of significance $(p)$ value is greater than 0.05 it is considered to be non-significant. All these statistical analyses were performed using SPSS statistical (Version 13 for Windows XP, SPSS, and Chicago, IL, USA). Multidimensional Scaling (MDS) and BrayCurtis similarity index was constructed based on macro faunal abundance and environmental parameters. Based on the groups obtained cluster analysis, species having the greatest contribution to this division were determined using similarity percentage program PAST (statistical Version 1.93 for Windows $\mathrm{XP})$.

\section{Results}

\subsection{Physico-chemical characteristics}

Water temperature, salinity and dissolved oxygen concentration showed a strong seasonal pattern (Figs 2-5). The maximum water temperature was recorded during premonsoon $29.8^{\circ} \mathrm{C}$ in southern seagrass stations and minimum $27.7^{\circ} \mathrm{C}$ during the monsoon at mangrove sites. The highest salinity 35.4 was recorded during premonsoon at mangrove sites and lowest 27 during the monsoon at southern seagrass stations. Conversely, the lowest DO values were recorded during post-monsoon in mangrove sites and the highest DO during the monsoon at southern seagrass stations. In general, the highest $\mathrm{pH} 8.5$ was recorded during premonsoon in mangroves and lowest $\mathrm{pH} 7.4$ during monsoon at the northern seagrass regions respectively.

In nutrients, maximum surface silicates $9 \mu \mathrm{g} / \mathrm{L}$ were recorded during premonsoon at southern seagrass stations and minimum $0.6 \mu \mathrm{g} / \mathrm{L}$ at the northern seagrass stations (Fig. 6), relatively higher phosphate intensity $4.6 \mu \mathrm{g} / \mathrm{L}$ was recorded during post monsoon and low $0.7 \mu \mathrm{g} / \mathrm{L}$ during premonsoon at southern seagrass stations (Fig. 7). The maximum nitrite $3.7 \mu \mathrm{g} / \mathrm{L}$ were recorded during premonsoon at northern seagrass stations and minimum $0.2 \mu \mathrm{g} / \mathrm{L}$ during premonsoon at mangrove sites (Fig. 8). Conversely, the lowest nitrate level $0.3 \mu \mathrm{g} / \mathrm{L}$ were recorded during monsoon in northern seagrass station and the highest value $3.6 \mu \mathrm{g} / \mathrm{L}$ during premonsoon at the mangrove site (Fig. 9).

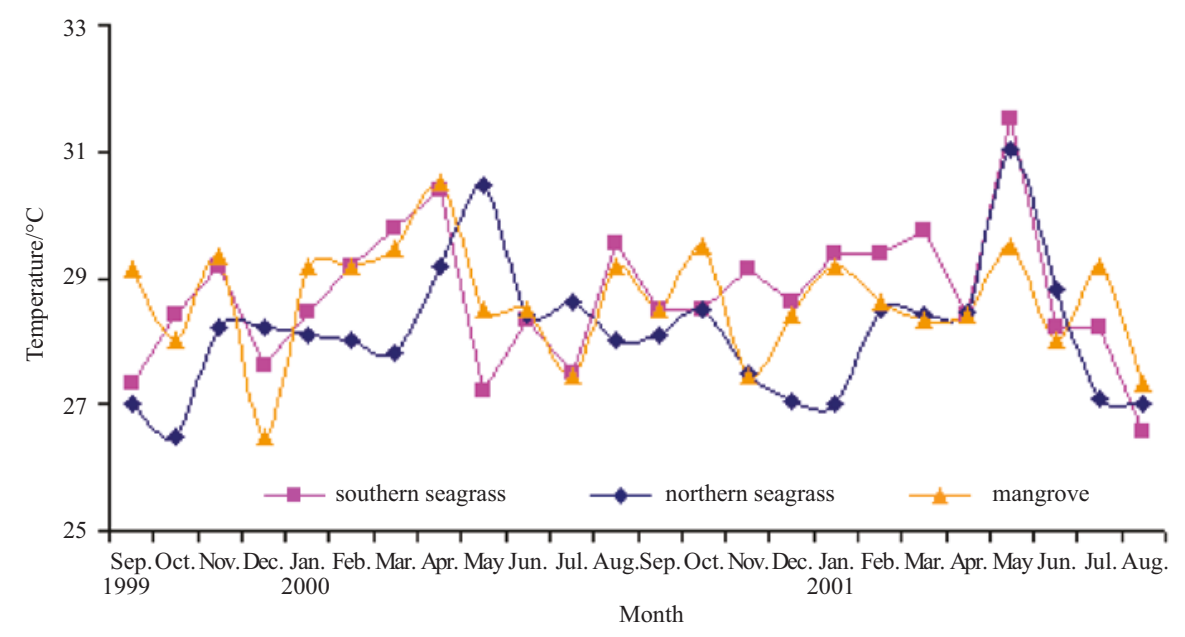

Fig.2. Monthly variations in water temperature at the three study regions. 


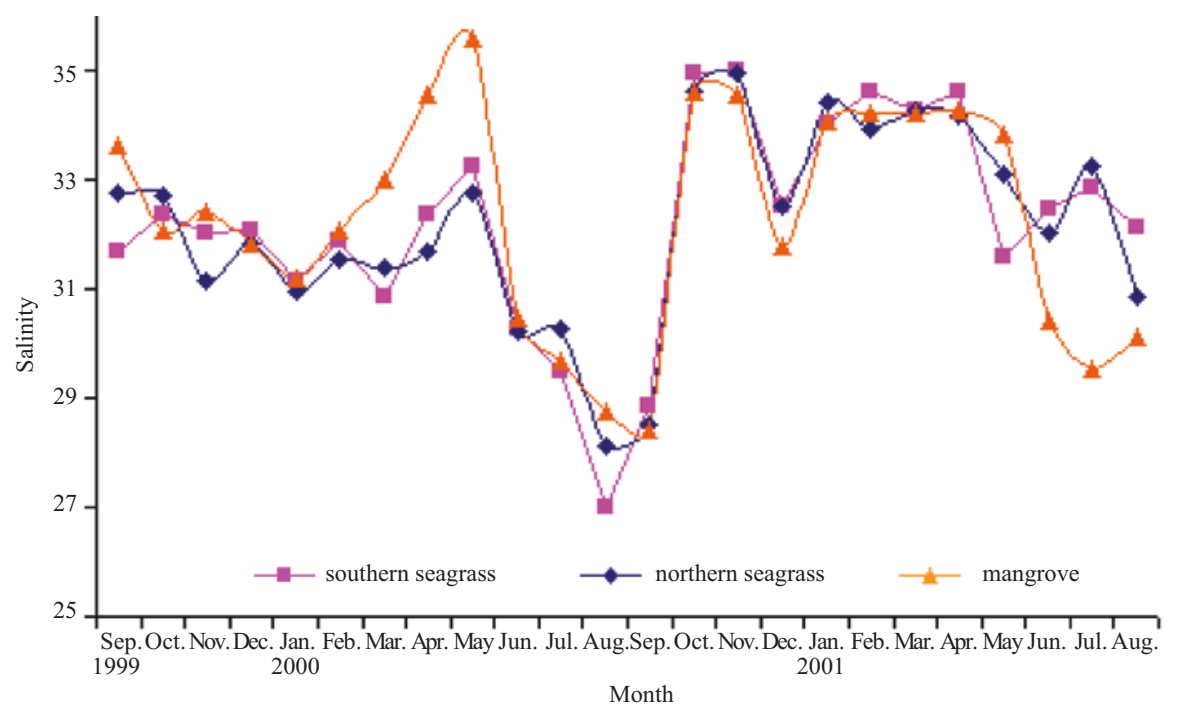

Fig.3. Monthly variations in salinity at the three study regions.

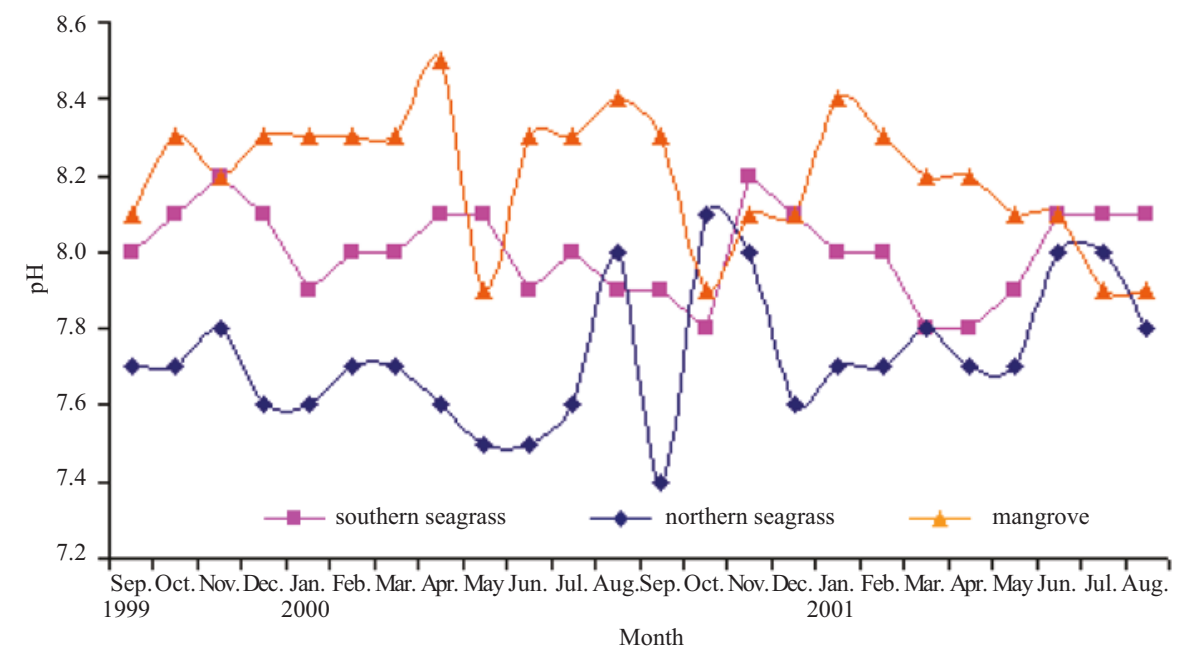

Fig.4. Monthly variations in $\mathrm{pH}$ at the three study regions.

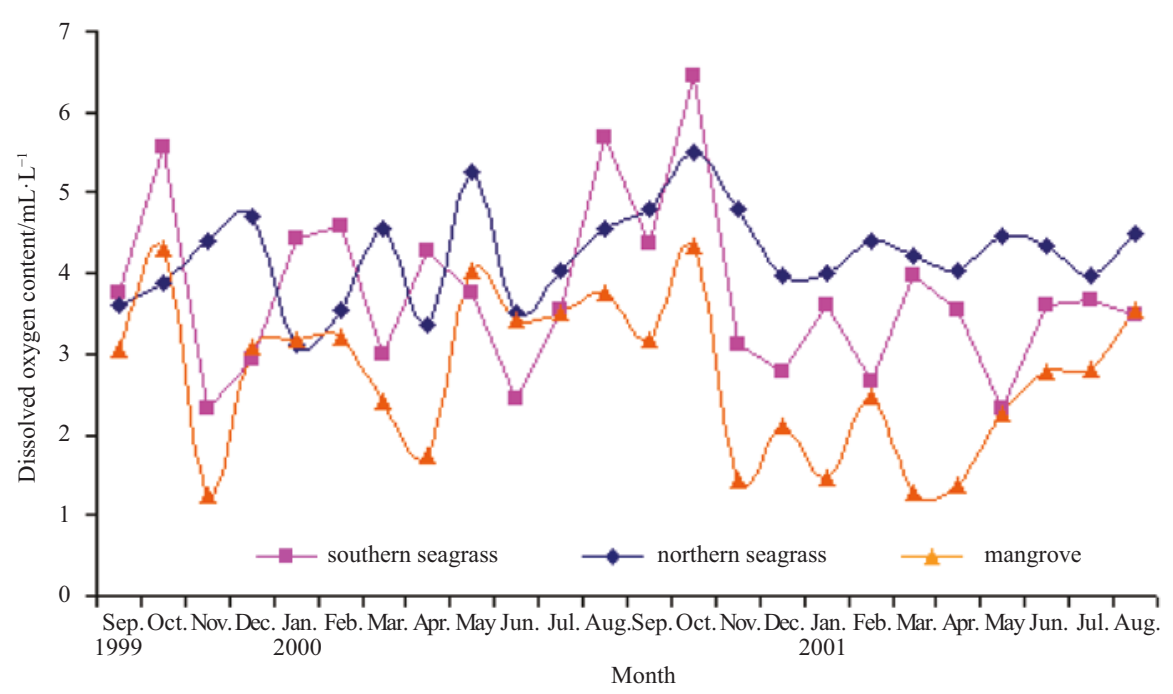

Fig.5. Monthly variations in dissolved oxygen at the three study regions. 


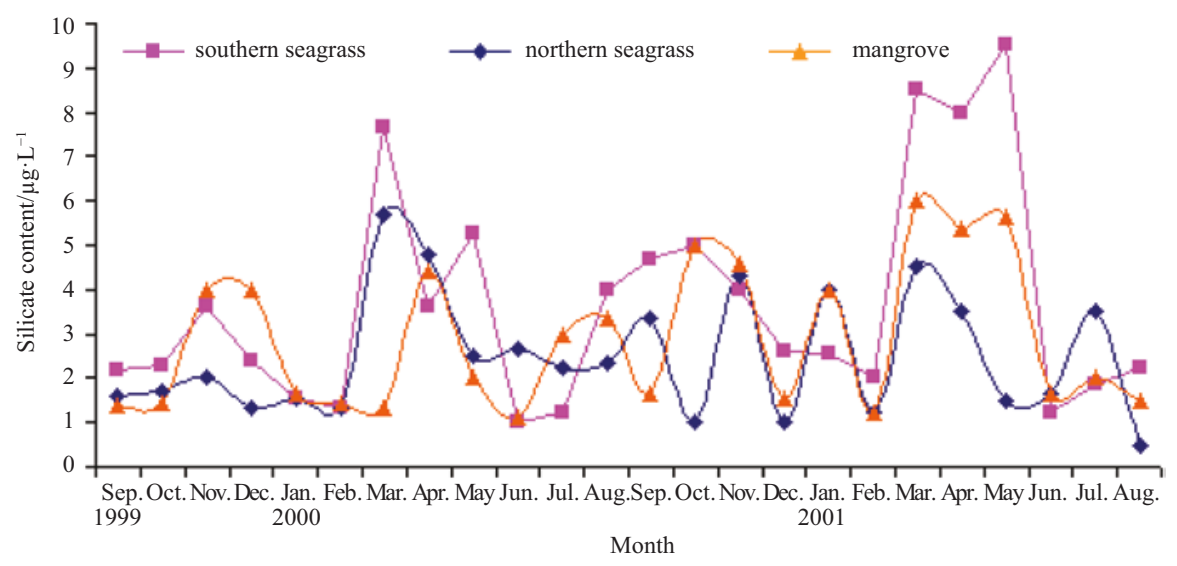

Fig.6. Monthly variations in silicates at the three study regions.

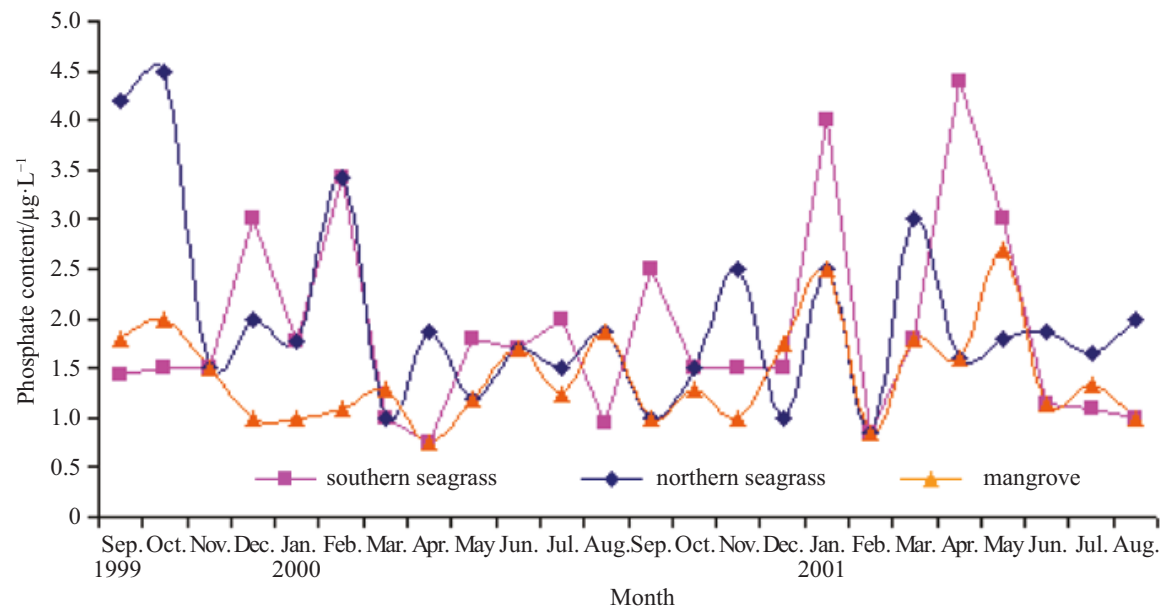

Fig.7. Monthly variations in phosphates at the three study regions.

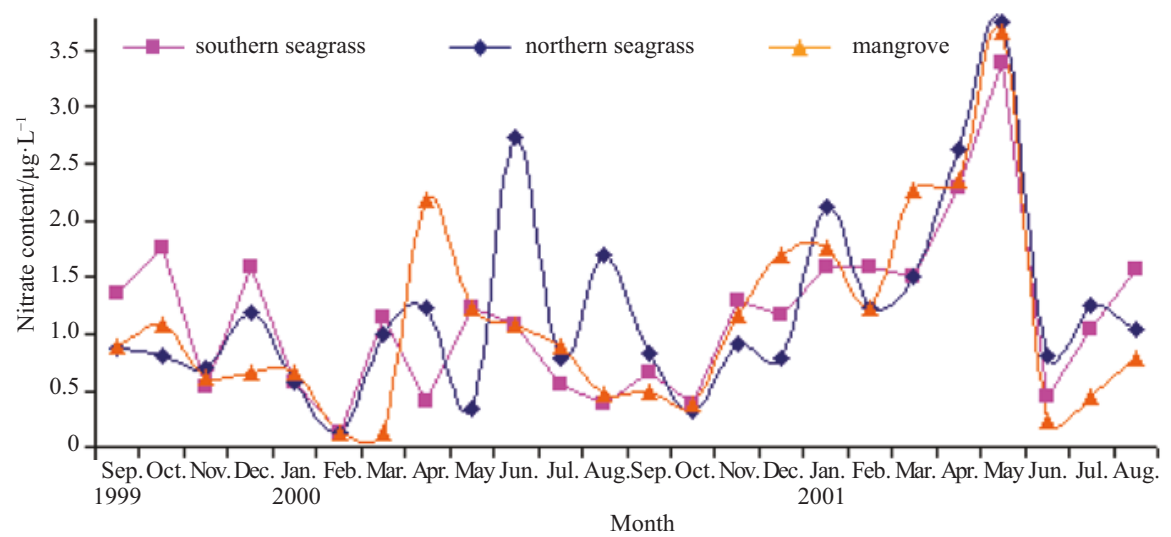

Fig.8. Monthly variations in nitrate at the three study regions.

\subsection{Sediment composition}

Table 2 shows the sediment composition varied greatly among sites between seagrass and mangrove stations. The substratum of the seagrass and mangroves was predominated by sand followed by clay and silt in comparatively smaller proportions. The highest value of sand was observed in monsoon and post monsoon seasons (95\%-96\%) and slightly less in the pre monsoon (90\%). Silt percentage remained almost the same during all seasons, showing little variations. In both years, comparatively higher percentage of clay was observed in pre monsoon season. Monsoon showed slightly lower values of clay in mangrove area. Organic carbon varied from 0.30 to 1.95 at seagrass ecosystem and relatively higher organic carbon $(2.1 \%-4.5 \%)$ at mangrove area (Fig. 10). 


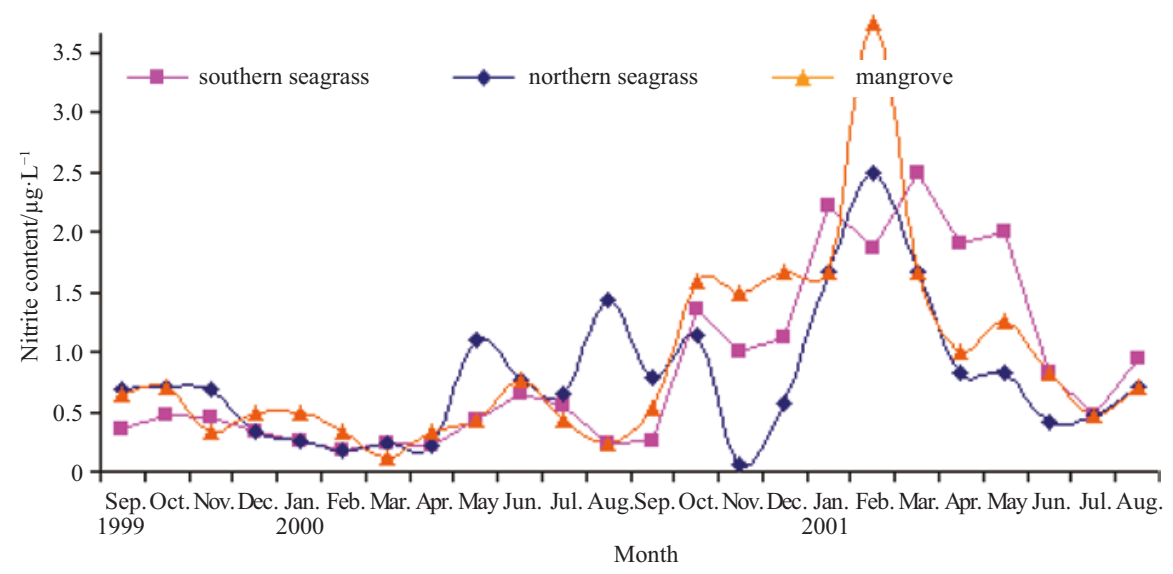

Fig.9. Monthly variations in nitrite at the three study regions.

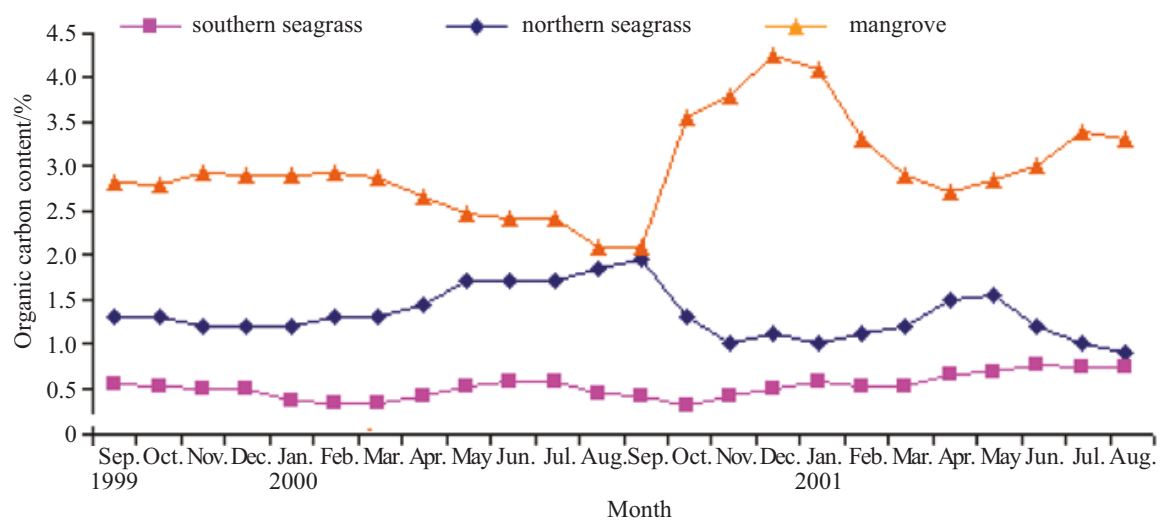

Fig.10. Sediment organic carbon content at three study areas.

Table 2. Season wise sand/silt/clay fraction at the three areas

\begin{tabular}{|c|c|c|c|c|c|c|c|c|c|c|}
\hline \multirow{2}{*}{ Year } & \multirow{2}{*}{ Season } & \multicolumn{3}{|c|}{ Sand $/ \%$} & \multicolumn{3}{|c|}{ Silt/\% } & \multicolumn{3}{|c|}{ Clay/\% } \\
\hline & & SSR & NSR & Mang & SSR & NSR & Mang & SSR & NSR & Mang \\
\hline 1999 & postmonsoon & 94 & 96 & 82 & 2 & 3 & 3 & 4 & 1 & 15 \\
\hline \multirow[t]{3}{*}{2000} & premonsoon & 90 & 94 & 78 & 2 & 4 & 6 & 8 & 2 & 16 \\
\hline & monsoon & 91 & 91 & 86 & 3 & 1 & 1 & 6 & 8 & 13 \\
\hline & postmonsoon & 95 & 96 & 82 & 1 & 1 & 4 & 4 & 3 & 14 \\
\hline \multirow[t]{2}{*}{2001} & premonsoon & 90 & 92 & 83 & 3 & 2 & 0 & 7 & 6 & 17 \\
\hline & monsoon & 95 & 96.5 & 85 & 1.5 & 1.5 & 1 & 3.5 & 2 & 14 \\
\hline
\end{tabular}

Notes: SSR represents southern seagrass region, NSR northern seagrass region, and Mang mangrove zone.

\subsection{Macrofaunal abundance and biomass}

The macrobenthic fauna in the study area showed great diversity in seagrass stations and low diversity in mangrove sites (Fig. 11). Distinct differences were found in the population density as well as the qualitative composition of the various taxa in different areas. A total of 160 macrobenthic species from eight major groups represented the macrofauna of the Minicoy Island (Table 3). Of the identified taxa molluscs 70 (gastropods $41.46 \%$, bivalves $7.5 \%)$, polychaetes 27 (16.88\%), crustaceans 30 (18.75\%), echinoderms $11(6.88 \%)$ and remaining others. Among molluscs gastropods constitute the bulk forming 51 species under 27 genera. A total of 70 species of molluscs including seven species of soft molluscs were recorded from seagrass bed for the first time on Minicoy Island, so far none of the previous studies from Indian seagrass beds reported any information on soft molluscs. The maximum proportion $(84.28 \%)$ of molluscs was observed in the southern seagrass region (SSR) and the minimum (12.85\%) in mangrove sites. The next most abundant fauna were polychaetes with maximum relative abundance $(74.07 \%)$ at SSR and minimum $(40.79 \%)$ at a northern seagrass region (NSR). Polychaetes were totally absent in mangrove ecosystem. Abundance of macrobenthic organisms, particularly mollusc, polychaetes and crustaceans were also high in SSR and most of them preferred sand dominating sediment. The average total biomass was maximum at Sta. $1\left(181.44 \mathrm{~g} / \mathrm{m}^{2}\right)$ and least average biomass was at Sta. $4\left(56.98 \mathrm{~g} / \mathrm{m}^{2}\right)$ with least spatial variation at Sta. 5 (42.87\%) and maximum at Sta. 1 (79.92\%) in Table 4. In general, a marked seasonal variation in biomass values of 


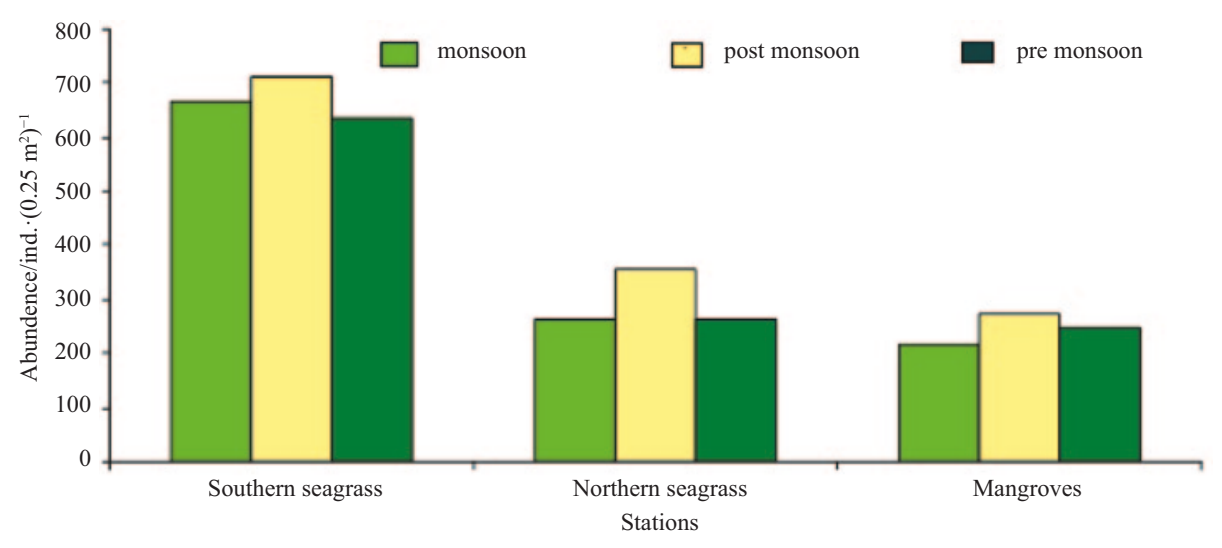

Fig.11. Average seasonal abundance in various study sites.

Table 3. Species wise contribution (\%) of numerical abundance to total abundance

\begin{tabular}{|c|c|c|c|c|c|c|}
\hline Species & Station 1 & Station 2 & Station 3 & Station 4 & Station 5 & Station 6 \\
\hline \multicolumn{7}{|l|}{ Gastropods } \\
\hline Punctacteon amakusaensis & 0.03 & 0.11 & 0.22 & 0.00 & 0.00 & 0.00 \\
\hline Marania lirata & 0.14 & 0.09 & 0.00 & 0.00 & 0.00 & 0.00 \\
\hline Cerithium corallium & 22.58 & 29.76 & 3.94 & 3.85 & 10.62 & 11.61 \\
\hline Cerithium alveolum & 3.63 & 3.12 & 1.22 & 1.67 & 0.00 & 0.00 \\
\hline Cerithium dialeucum & 0.31 & 0.60 & 0.50 & 0.29 & 0.00 & 0.00 \\
\hline Cerithium rarimaculatum & 0.03 & 0.07 & 0.00 & 0.00 & 0.43 & 0.19 \\
\hline Cerithium scabridum & 13.89 & 19.98 & 6.61 & 8.10 & 0.50 & 0.77 \\
\hline Cerithium rostratum & 0.39 & 1.60 & 7.05 & 4.37 & 0.00 & 0.00 \\
\hline Cerithium nesioticum & 0.82 & 5.79 & 3.00 & 4.02 & 0.00 & 0.00 \\
\hline Clypeomorus corallium & 0.06 & 0.02 & 0.00 & 0.00 & 0.00 & 0.00 \\
\hline Rhinoclavis sinensis & 0.06 & 0.20 & 0.06 & 0.23 & 0.00 & 0.00 \\
\hline Pyrene sp. & 2.25 & 1.87 & 11.83 & 12.70 & 0.93 & 0.38 \\
\hline Pyrene vulpecula & 0.65 & 0.47 & 1.50 & 0.46 & 0.00 & 0.00 \\
\hline Metanachis marquesa & 0.39 & 0.27 & 1.00 & 3.85 & 0.00 & 0.00 \\
\hline Conus catus & 0.14 & 0.38 & 0.50 & 0.06 & 0.00 & 0.00 \\
\hline Conus ebraeus & 0.17 & 0.02 & 0.00 & 0.00 & 0.00 & 0.00 \\
\hline Coralliophila costularis & 0.51 & 0.80 & 0.17 & 0.23 & 0.00 & 0.00 \\
\hline Cyprea annulus & 0.08 & 0.04 & 0.00 & 0.00 & 0.00 & 0.00 \\
\hline Cyprea arabica & 0.08 & 0.07 & 0.00 & 0.00 & 0.00 & 0.00 \\
\hline Cyprea moneta & 0.51 & 0.27 & 0.33 & 0.00 & 0.00 & 0.00 \\
\hline Cyprea teres & 0.11 & 0.04 & 0.00 & 0.00 & 0.00 & 0.00 \\
\hline Cyprea tigris & 0.08 & 0.07 & 0.00 & 0.00 & 0.00 & 0.00 \\
\hline Mazescala japonica & 0.00 & 0.02 & 0.00 & 0.00 & 0.00 & 0.00 \\
\hline Niso heizensis & 0.00 & 0.36 & 1.50 & 0.46 & 0.00 & 0.00 \\
\hline Persternia pilsbryi & 0.08 & 0.00 & 0.00 & 0.00 & 0.00 & 0.00 \\
\hline Littorina undulata & 0.28 & 1.36 & 1.33 & 0.00 & 59.37 & 66.52 \\
\hline Strigatella litterata & 0.00 & 0.02 & 0.00 & 0.00 & 0.00 & 0.00 \\
\hline Drupella sp. & 0.03 & 0.04 & 0.00 & 0.06 & 0.00 & 0.00 \\
\hline Nassarius distortus & 0.00 & 0.02 & 0.00 & 0.00 & 0.00 & 0.00 \\
\hline Niotha stigmaria & 0.03 & 0.40 & 0.17 & 0.52 & 0.00 & 0.00 \\
\hline Zeuxis sp. & 0.34 & 0.00 & 0.00 & 0.00 & 0.00 & 0.00 \\
\hline Polinices flemengium & 0.25 & 0.00 & 1.44 & 0.11 & 0.00 & 0.00 \\
\hline Natica rufa & 0.06 & 0.07 & 0.00 & 0.00 & 0.00 & 0.00 \\
\hline
\end{tabular}


continued from Table 3

\begin{tabular}{|c|c|c|c|c|c|c|}
\hline Species & Station 1 & Station 2 & Station 3 & Station 4 & Station 5 & Station 6 \\
\hline Smaragdia viridis & 2.47 & 2.83 & 5.00 & 2.53 & 0.43 & 0.19 \\
\hline Smaragdia soverbiana & 7.84 & 6.97 & 2.72 & 2.59 & 0.14 & 0.64 \\
\hline Vittina variegata & 0.00 & 0.78 & 0.50 & 0.06 & 0.00 & 0.00 \\
\hline Terebralia palustris & 0.00 & 0.00 & 0.00 & 0.00 & 18.03 & 14.99 \\
\hline Agatha virgo & 0.00 & 0.00 & 0.00 & 0.00 & 0.00 & 0.00 \\
\hline Agatha lepidule & 0.00 & 0.00 & 0.00 & 0.00 & 0.00 & 0.00 \\
\hline Pyrgulina pupula & 0.00 & 0.09 & 0.72 & 0.23 & 0.00 & 0.00 \\
\hline Cymatium neobaricum & 0.00 & 0.04 & 0.11 & 0.00 & 0.00 & 0.00 \\
\hline Cymatriton nicobaricum & 0.00 & 0.00 & 0.11 & 0.00 & 0.00 & 0.00 \\
\hline Cinctiscala sp. & 0.39 & 0.16 & 0.00 & 0.00 & 0.00 & 0.00 \\
\hline Decorifer insignis & 0.11 & 0.07 & 0.00 & 0.00 & 0.00 & 0.00 \\
\hline Casmeria ponderisai & 0.00 & 0.07 & 0.00 & 0.06 & 0.00 & 0.00 \\
\hline Strombus canarium & 0.20 & 0.02 & 0.17 & 0.00 & 0.00 & 0.00 \\
\hline Strombus mutabilis & 0.20 & 0.20 & 0.28 & 0.00 & 0.00 & 0.00 \\
\hline Cinguloterebra hedleyana & 0.14 & 0.49 & 0.33 & 0.57 & 0.00 & 0.00 \\
\hline Margarites helicina & 0.39 & 0.38 & 0.00 & 0.00 & 0.57 & 0.70 \\
\hline Truncatella pfeifferi & 0.00 & 0.00 & 0.00 & 0.00 & 0.00 & 0.00 \\
\hline Unidentified 129 & 0.51 & 0.18 & 0.61 & 0.34 & 0.00 & 0.00 \\
\hline \multicolumn{7}{|l|}{ Soft molluscs } \\
\hline Dolabella rumphii & 0.34 & 0.22 & 0.00 & 0.00 & 0.00 & 0.00 \\
\hline Polycera sp. & 0.08 & 0.09 & 0.00 & 0.00 & 0.00 & 0.00 \\
\hline Gymnodoris ceylonica & 0.03 & 0.02 & 0.00 & 0.00 & 0.00 & 0.00 \\
\hline Elysia sp. & 0.31 & 0.09 & 0.00 & 0.00 & 0.00 & 0.00 \\
\hline Smargdinella canaliculata & 0.20 & 0.13 & 0.00 & 0.00 & 0.00 & 0.00 \\
\hline diala lauta & 0.00 & 0.00 & 0.00 & 0.00 & 0.00 & 0.00 \\
\hline Dolabrifera dolabrifera & 0.00 & 0.00 & 0.00 & 0.00 & 0.00 & 0.00 \\
\hline \multicolumn{7}{|l|}{ Bivalves } \\
\hline Lunulicardia auricula & 0.08 & 0.00 & 0.00 & 0.00 & 0.00 & 0.00 \\
\hline Cardium asiaticum & 0.37 & 0.04 & 0.17 & 0.00 & 0.00 & 0.00 \\
\hline Corculum impressum & 0.11 & 0.04 & 0.00 & 0.00 & 0.00 & 0.00 \\
\hline Ctena delicatula & 0.42 & 0.33 & 0.44 & 0.00 & 0.00 & 0.00 \\
\hline Mactra cuneata & 0.06 & 0.02 & 0.00 & 0.00 & 0.00 & 0.00 \\
\hline Myadoropsis brevispinious & 0.17 & 0.20 & 0.00 & 0.17 & 0.00 & 0.00 \\
\hline Lithophaga nigra & 0.14 & 0.13 & 0.00 & 0.00 & 0.00 & 0.00 \\
\hline Modiolus metcalfei & 0.06 & 0.09 & 0.06 & 0.11 & 0.00 & 0.00 \\
\hline Pinna muricata & 0.34 & 0.29 & 0.00 & 0.00 & 0.00 & 0.00 \\
\hline Tellina palatum & 0.14 & 0.07 & 1.44 & 1.38 & 0.00 & 0.00 \\
\hline Gafrarium divarticatum & 8.74 & 4.43 & 18.93 & 25.92 & 0.00 & 0.00 \\
\hline Periglypta puerpura & 0.08 & 0.27 & 0.00 & 0.00 & 0.00 & 0.00 \\
\hline \multicolumn{7}{|l|}{ Worms } \\
\hline Baseodiscus delineatus & 0.37 & 0.25 & 1.22 & 3.79 & 0.00 & 0.00 \\
\hline Golfingia hespera & 0.17 & 0.20 & 0.67 & 0.34 & 0.00 & 0.00 \\
\hline Phascolosoma nigrescens & 0.08 & 0.07 & 2.22 & 2.82 & 0.00 & 0.00 \\
\hline Siphonosoma australe & 0.08 & 0.00 & 0.33 & 0.34 & 0.00 & 0.00 \\
\hline Sipunculus indicus & 0.14 & 0.09 & 0.61 & 0.40 & 0.00 & 0.00 \\
\hline Siboglinum fiordicum & 1.15 & 0.29 & 1.33 & 2.13 & 0.00 & 0.00 \\
\hline Hoplonemertean sp. & 0.11 & 0.16 & 0.22 & 0.34 & 0.00 & 0.00 \\
\hline \multicolumn{7}{|l|}{ Polychaete worms } \\
\hline Scoloplos sp. & 0.06 & 0.02 & 0.00 & 0.00 & 0.00 & 0.00 \\
\hline Eurythoe complanata & 0.28 & 0.02 & 0.56 & 1.84 & 0.00 & 0.00 \\
\hline
\end{tabular}


continued from Table 3

\begin{tabular}{|c|c|c|c|c|c|c|}
\hline Species & Station 1 & Station 2 & Station 3 & Station 4 & Station 5 & Station 6 \\
\hline Eurythoe mathaii & 0.14 & 0.07 & 2.39 & 0.00 & 0.00 & 0.00 \\
\hline Notopygos variabilis & 0.20 & 0.04 & 0.00 & 0.23 & 0.00 & 0.00 \\
\hline Notomastes latericeus & 0.17 & 0.13 & 0.39 & 1.21 & 0.00 & 0.00 \\
\hline Cirratulus sp. & 0.14 & 0.09 & 0.00 & 0.00 & 0.00 & 0.00 \\
\hline Marphysa macintoshi & 0.03 & 0.04 & 0.33 & 0.52 & 0.00 & 0.00 \\
\hline Nematonereis unicornis & 0.11 & 0.00 & 0.11 & 0.00 & 0.00 & 0.00 \\
\hline Glycera convoluta & 0.28 & 0.16 & 0.00 & 0.00 & 0.00 & 0.00 \\
\hline Glycera lancadivae & 1.46 & 0.53 & 0.22 & 0.11 & 0.00 & 0.00 \\
\hline Glycera subaena & 0.22 & 0.13 & 0.50 & 0.00 & 0.00 & 0.00 \\
\hline Glycera tesselata & 0.22 & 0.09 & 1.89 & 3.68 & 0.00 & 0.00 \\
\hline Glycera sp. & 0.06 & 0.00 & 0.33 & 0.29 & 0.00 & 0.00 \\
\hline Goniada emerita & 0.25 & 0.20 & 0.17 & 0.34 & 0.00 & 0.00 \\
\hline Nephtys dibranchus & 0.20 & 0.07 & 0.06 & 0.00 & 0.00 & 0.00 \\
\hline Nephtys hombergii & 1.38 & 0.25 & 0.11 & 0.00 & 0.00 & 0.00 \\
\hline Nephtys inermis & 0.25 & 0.11 & 0.22 & 0.00 & 0.00 & 0.00 \\
\hline Ceratonereis erythraensis & 0.22 & 0.09 & 0.56 & 0.17 & 0.00 & 0.00 \\
\hline Nereis kauderni & 0.28 & 0.04 & 0.00 & 0.17 & 0.00 & 0.00 \\
\hline Nereis trifasciata & 1.07 & 0.42 & 0.50 & 0.00 & 0.00 & 0.00 \\
\hline Arabella iricolor iricolor & 0.17 & 0.09 & 0.11 & 0.00 & 0.00 & 0.00 \\
\hline Oenone fulgida & 0.17 & 0.07 & 0.00 & 0.00 & 0.00 & 0.00 \\
\hline Armandia sp. & 0.11 & 0.09 & 0.00 & 0.00 & 0.00 & 0.00 \\
\hline Megalomma sp. & 0.06 & 0.04 & 0.00 & 0.00 & 0.00 & 0.00 \\
\hline Syllis cornuta & 0.20 & 0.13 & 0.22 & 0.17 & 0.00 & 0.00 \\
\hline Syllis gracilis & 0.11 & 0.13 & 0.22 & 0.00 & 0.00 & 0.00 \\
\hline Eupolymna nebulosa & 0.11 & 0.07 & 0.39 & 0.52 & 0.00 & 0.00 \\
\hline \multicolumn{7}{|l|}{ Crab } \\
\hline Leptoduis sp. & 0.22 & 0.09 & 0.00 & 0.06 & 0.00 & 0.00 \\
\hline Polydectus cuculifer & 0.06 & 0.02 & 0.00 & 0.00 & 0.00 & 0.00 \\
\hline Megalopa larva & 0.31 & 0.31 & 0.00 & 0.00 & 0.00 & 0.00 \\
\hline Calappa hepatica & 0.82 & 0.25 & 0.00 & 0.34 & 0.00 & 0.00 \\
\hline Diogene sp. & 0.03 & 0.02 & 0.06 & 0.00 & 0.00 & 0.00 \\
\hline Cardiosoma carnifex & 0.00 & 0.02 & 0.11 & 0.00 & 0.07 & 0.00 \\
\hline Pachygrapsus plicatus & 0.17 & 0.04 & 0.00 & 0.11 & 0.00 & 0.00 \\
\hline Illyograpsus paludicola & 0.00 & 0.02 & 0.00 & 0.00 & 0.00 & 0.06 \\
\hline Plagusia sp. & 0.14 & 0.00 & 0.00 & 0.00 & 0.00 & 0.00 \\
\hline Grapsus sp. & 0.25 & 0.04 & 0.06 & 0.00 & 0.00 & 0.00 \\
\hline Eriphis sp. & 0.20 & 0.07 & 0.39 & 0.17 & 0.00 & 0.00 \\
\hline Uca tetragonon & 0.00 & 0.02 & 0.00 & 0.00 & 0.14 & 0.26 \\
\hline Macrophthalmus boscii & 0.93 & 0.09 & 0.11 & 0.80 & 0.00 & 0.00 \\
\hline Uca inversa inversa & 0.00 & 0.00 & 0.00 & 0.06 & 0.07 & 0.13 \\
\hline Tylodipax desigardi & 0.11 & 0.07 & 0.00 & 0.29 & 0.14 & 0.00 \\
\hline Pilumnus hirtellus & 0.45 & 0.09 & 0.39 & 0.29 & 0.00 & 0.00 \\
\hline Pinnotheres pisum & 0.17 & 0.04 & 0.06 & 0.06 & 0.00 & 0.00 \\
\hline Pinnotheres pinnotheres & 0.25 & 0.47 & 0.72 & 0.63 & 0.00 & 0.00 \\
\hline Thalamita crenata & 0.76 & 0.49 & 0.94 & 0.34 & 0.14 & 0.00 \\
\hline scylla serrata & 0.00 & 0.00 & 0.00 & 0.00 & 0.43 & 0.19 \\
\hline Portunus orbitosinus & 0.03 & 0.07 & 0.00 & 0.00 & 0.00 & 0.00 \\
\hline Macropipus corrugatus & 0.00 & 0.00 & 0.00 & 0.00 & 0.00 & 0.00 \\
\hline Etisus splendidus & 0.22 & 0.02 & 0.00 & 0.00 & 0.00 & 0.00 \\
\hline Actaeodes tomentosus & 0.28 & 0.11 & 0.44 & 0.00 & 0.00 & 0.00 \\
\hline
\end{tabular}


continued from Table 3

\begin{tabular}{|c|c|c|c|c|c|c|}
\hline Species & Stationl & Station 2 & Station 3 & Station 4 & Station 5 & Station 6 \\
\hline \multicolumn{7}{|l|}{ Prawns and shrimps } \\
\hline Alpheopsis equalis & 0.25 & 0.09 & 0.06 & 0.11 & 0.00 & 0.00 \\
\hline Alpheus lottini & 0.00 & 0.00 & 0.00 & 0.00 & 0.00 & 0.00 \\
\hline Alpheus sp. & 0.06 & 0.02 & 0.00 & 0.00 & 0.00 & 0.00 \\
\hline Metabetaeus minutus & 0.06 & 0.07 & 0.00 & 0.00 & 0.00 & 0.00 \\
\hline Nikoides maldivensis & 0.00 & 0.09 & 0.00 & 0.00 & 1.57 & 0.89 \\
\hline \multicolumn{7}{|l|}{ Amphipods } \\
\hline Cymadusa imbroglio & 2.42 & 0.22 & 4.22 & 1.09 & 0.00 & 0.00 \\
\hline Maera pacifica & 4.39 & 3.43 & 2.33 & 1.09 & 0.00 & 0.00 \\
\hline Mallacoota insignis & 0.28 & 0.31 & 0.00 & 0.00 & 0.00 & 0.00 \\
\hline Stenothoe kaia & 1.10 & 0.27 & 0.00 & 0.06 & 0.00 & 0.00 \\
\hline \multicolumn{7}{|l|}{ Isopods } \\
\hline Cirolana sp. & 0.03 & 0.11 & 0.00 & 0.00 & 0.00 & 0.00 \\
\hline Seychellana expansa & 0.17 & 0.09 & 0.17 & 0.00 & 0.00 & 0.00 \\
\hline Accalathura borradailei & 0.14 & 0.11 & 0.00 & 0.00 & 0.00 & 0.00 \\
\hline Paracilicacea setosa & 0.45 & 0.16 & 0.00 & 0.00 & 0.00 & 0.00 \\
\hline Paraleptosphaeroma indica & 0.14 & 0.09 & 0.00 & 0.00 & 0.00 & 0.00 \\
\hline \multicolumn{7}{|l|}{ Stomatopods } \\
\hline Gonodactylus.of.smithii & 0.06 & 0.04 & 0.00 & 0.00 & 0.00 & 0.00 \\
\hline Apseudus sp. & 0.03 & 0.00 & 0.00 & 0.00 & 5.92 & 2.42 \\
\hline Paratanaeidae sp. & 0.67 & 0.22 & 0.00 & 0.00 & 0.50 & 0.06 \\
\hline Paranebalia sp. & 0.11 & 0.09 & 0.00 & 0.00 & 0.00 & 0.00 \\
\hline Siriella brevicaudata & 0.14 & 0.18 & 0.00 & 0.00 & 0.00 & 0.00 \\
\hline \multicolumn{7}{|l|}{ Echinoderms } \\
\hline Linckia multifora & 0.08 & 0.00 & 0.00 & 0.00 & 0.00 & 0.00 \\
\hline Ophiactis savignyi & 0.25 & 0.13 & 0.00 & 0.11 & 0.00 & 0.00 \\
\hline Ophicornella sexadia & 0.62 & 0.31 & 0.11 & 0.00 & 0.00 & 0.00 \\
\hline Ophiocoma scolopendrina & 0.34 & 0.11 & 0.00 & 0.00 & 0.00 & 0.00 \\
\hline Astropyga radiata & 0.03 & 0.00 & 0.00 & 0.00 & 0.00 & 0.00 \\
\hline Echinometra mathaei & 0.17 & 0.07 & 0.00 & 0.00 & 0.00 & 0.00 \\
\hline Echinoneus cyclostomus & 0.08 & 0.11 & 0.00 & 0.00 & 0.00 & 0.00 \\
\hline Salmacis bicolor & 0.11 & 0.02 & 0.00 & 0.00 & 0.00 & 0.00 \\
\hline Bohadschia subruba & 0.06 & 0.02 & 0.00 & 0.00 & 0.00 & 0.00 \\
\hline Holothuria nobilis & 0.03 & 0.04 & 0.00 & 0.00 & 0.00 & 0.00 \\
\hline Holothuria scabra & 0.06 & 0.00 & 0.00 & 0.00 & 0.00 & 0.00 \\
\hline \multicolumn{7}{|l|}{ Sponges } \\
\hline Aaptos cf. chromis & 0.22 & 0.27 & 0.00 & 0.00 & 0.00 & 0.00 \\
\hline Cinachyrella voeltzkowii & 0.11 & 0.07 & 0.00 & 0.00 & 0.00 & 0.00 \\
\hline
\end{tabular}

Table 4. Station wise average biomass of major groups of benthos $\left(\mathrm{g} / \mathrm{m}^{2}\right)$

\begin{tabular}{|c|c|c|c|c|c|c|}
\hline \multirow{2}{*}{ Major groups } & \multicolumn{2}{|c|}{ Area 1} & \multicolumn{2}{|c|}{ Area 2} & \multicolumn{2}{|c|}{ Area 3} \\
\hline & Station 1 & Station 2 & Station 3 & Station 4 & Station 5 & Station 6 \\
\hline Gastropods & 167.93 & 150.29 & 33.89 & 31.96 & 115.48 & 98.53 \\
\hline Bivalves & 11.15 & 13.3 & 42.32 & 22.01 & 0 & 0 \\
\hline Worms (including polychaetes) & 0.73 & 0.4 & 1.2 & 1.35 & 0 & 0 \\
\hline Crabs & 0.65 & 0.67 & 1.11 & 1.09 & 2.23 & 1.59 \\
\hline Other crustaceans & 0.3 & 0.23 & 0.17 & 0.03 & 0.62 & 0.97 \\
\hline Echinoderms & 0.5 & 0.37 & 0.01 & 0.02 & 0 & 0 \\
\hline Sponges & 0.18 & 0.35 & 0 & 0 & 0 & 0 \\
\hline Total & 181.44 & 165.61 & 78.7 & 56.46 & 118.33 & 101.09 \\
\hline
\end{tabular}


the benthic fauna was observed. From the season wise analysis, it was found that post-monsoon season contributed a major share of total biomass followed by monsoon in all three areas and the lowest total biomass was observed in pre-monsoon season in all three areas.

\subsection{Dominant taxa}

The three dominant species representing $25.92 \%-66.52 \%$ of the macrofaunal community; among these species, the gastropods Littorina undulata $(66.52 \%)$ were dominant on the mangrove site at Sta. 6, Cerithium corallium $(29.76 \%)$ in SSR at Sta. 2 and bivalve G. divarticatum (25.92\%) in NSR at Sta. 4. However the faunal composition decreased at the mangroves in Minicoy Island. In Sta. 3 at NSR polychaetes Eurythoe mathaii (2.39\%) were dominated and E. complanata $(1.84 \%)$ at Sta. 4 . Their abundances at mangrove sites declined. Amphipod species dominated in SSR at Sta. 1, Maera pacifica contribute $4.39 \%$, Amphipods completely absent from mangrove sites.

\subsection{Species diversity indices}

Species dominance in the study area was least at Sta. $2(0.47)$ and maximum at Sta. 3 (0.803). The pattern of distribution was same as that of diversity and concentration with two modal values, one at Sta. 1 and the other at Sta. 3 (Table 5). Species evenness was least at Sta. 2 (0.692) and maximum at Sta. 3 (1.66). Values of $H^{\prime}$ varied from 1.45 to 3.64, the highest $H^{\prime}$ value was also observed in the southern sea-grass region at Sta. 1 and least in mangrove site at Sta. 6. Species richness index values varied from 4.19 to 26.17 , based on average diversity indices computed for each station, it was noticed that there was a steady decrease in the species richness index from Sta. 1 to Sta. 6 with a gradual increase in spatial variation even though overall variation was less $(<36.26 \%)$ except at Sta. 2 , where the coefficient of variation was the least for species richness (15.49\%).

\subsection{Macrofaunal composition}

\subsubsection{Southern seagrass ecosystem}

A total of 137 taxa was identified, amongst molluscs was the dominant group comprising gastropods $71 \%$ and bivalves $9 \%$ of the total final abundance. Of the 27 molluscs families identified in the entire area, 23 were observed at Sta. 2. All Cerithium spp. together contributed $90 \%$ of gastropods, among bivalves Cardium divarticum contributed $90 \%$ of individuals followed by $C$. asiaticum and Pinna maricata. Polychaete species like Glycera, Nereis and Nephtys were present in considerable number while the other species of this group were less. However crab Calappa hepatica formed the major share of crabs followed by Thalamita crenata. Carridean prawns formed $90 \%$ of other crustaceans, the major share contributed by Alpheopsis equalis and Alpheus sp. In the southern seagrass beds, amphipods flourished seasonally and they contributed $7 \%$ of total individuals, the major species involved Maera pacifica (4.39\%). Echinoderms contributed $2 \%$ of total individuals, of which major share was contributed by Ophiocornella sexadia and Ophicoma scolopendrina. Sponge Aaptos cf. chromis was found in almost all seagrass samples. In SSR, gastropods showed a highest seasonal average of $553 \mathrm{nos} /\left(0.25 \mathrm{~m}^{2}\right)$, bivalves, crabs and echinoderms showed highest abundance at the monsoon.

\subsubsection{Northern seagrass ecosystem}

Many species present at Stas 1 and 2 were not recorded from this site. In NSR most of the major groups like gastropods, bivalves and others, of the $53 \%$ gastropods, Cerithium spp. contributed $21 \%$ and Smaragdia spp. 7\%. Soft molluscs were not recorded from this station. Polychaetes present in good numbers,

Table 5. Community structure indices on the average

\begin{tabular}{|c|c|c|c|c|c|}
\hline Station & Richness index & Concentration index & Diversity index & Dominance index & Evenness index \\
\hline \multicolumn{6}{|l|}{ Station 1} \\
\hline $\bar{X}$ & 26.176 & 0.843 & 3.649 & 0.594 & 1.361 \\
\hline$\sigma$ & 5.674 & 0.067 & 0.504 & 0.349 & 0.674 \\
\hline C.V.\% & 21.678 & 7.929 & 13.948 & 58.77 & 49.559 \\
\hline \multicolumn{6}{|l|}{ Station 2} \\
\hline $\bar{X}$ & 20.45 & 0.702 & 2.778 & 0.470 & 0.692 \\
\hline$\sigma$ & 3.168 & 0.122 & 0.523 & 0.412 & 0.346 \\
\hline C.V.\% & 15.49 & 17.41 & 18.82 & 87.61 & 49.97 \\
\hline \multicolumn{6}{|l|}{ Station 3} \\
\hline $\bar{X}$ & 14.04 & 0.829 & 3.173 & 0.801 & 1.667 \\
\hline$\sigma$ & 3.242 & 0.078 & 0.438 & 0.105 & 0.500 \\
\hline C.V.\% & 23.07 & 9.388 & 13.79 & 13.05 & 29.97 \\
\hline \multicolumn{6}{|l|}{ Station 4} \\
\hline $\bar{X}$ & 12.52 & 0.813 & 3.039 & 0.772 & 1.629 \\
\hline$\sigma$ & 3.121 & 0.085 & 0.492 & 0.113 & 0.521 \\
\hline C.V.\% & 24.92 & 10.44 & 16.19 & 14.69 & 31.98 \\
\hline \multicolumn{6}{|l|}{ Station 5} \\
\hline $\bar{X}$ & 5.175 & 0.579 & 1.709 & 0.754 & 0.944 \\
\hline$\sigma$ & 1.321 & 0.106 & 0.354 & 0.156 & 0.306 \\
\hline C.V.\% & 25.53 & 18.27 & 20.71 & 20.71 & 32.38 \\
\hline \multicolumn{6}{|l|}{ Station 6} \\
\hline $\bar{X}$ & 4.198 & 0.500 & 1.453 & 0.641 & 0.853 \\
\hline$\sigma$ & 1.522 & 0.133 & 0.412 & 0.182 & 0.338 \\
\hline C.V.\% & 36.25 & 26.69 & 28.32 & 28.33 & 39.66 \\
\hline
\end{tabular}


Eurythoe mathaei contributed 25\% of total polychaetes from this region. Among crab population of $4 \%$, Thalamita crenata and Pilumnus hirtellus were dominated species. Crustaceans showed their highest average seasonal abundance during post monsoon. Only six species of bivalves were present at this station, of which G. divarticatum alone contributed $25.92 \%$ of individuals.

\subsubsection{Mangrove ecosystem}

Only nine species of gastropods were recorded, out of which Littorina undulata formed about $59 \%$ of total abundance. Bivalves, worms, echinoderms and sponges were not recorded from this station. Six species of crabs were found, out of which Scylla serrata showed the highest abundance. The total abundance of macrobenthos in mangrove areas showed the highest value during post-monsoon.

\subsection{Relationship between benthic fauna and environmental} parameters

Correlation analysis showed a meager relationship between benthic diversity indices and studied abiotic variables (Table 6). Among the independent variables analyzed, salinity indicated significantly a strong correlation with the benthic faunal dominance $(r=0.836, p<0.01)$ and species evenness $(r=0.689, p<0.05)$.
Significant correlation between dissolved oxygen (DO) and Shannon diversity $(r=0.740, p<0.05)$, among the physical environmental variables, phosphate had a high correlation $(r=0.855$, $p<0.01)$ with the Shannon diversity, evenness $(r=0.749, p<0.05)$ and richness $(r=0.689, p<0.05)$. Sediment characteristic clay shows a weak negative correlation between species dominance $(r=-0.673, p<0.05)$.

Three-way ANOVA applied on the benthic data showed that there was a significant difference between stations and abundance of various benthic groups. ANOVA analysis showed the significance of stations and abundances were generally significantly high at SSR and NSR, low in mangrove sites. They were relatively high at SSE during post monsoon (Three-way ANOVA; $F_{(44.65)}, n=126890, p<0.01$ ) (Table 7). Benthic abundances were generally higher at SSE and lower in mangrove sites. A general, higher gastropod abundance was found at SSR (Threeway ANOVA, $p<0.01$ ) and significant level between month and abundances was showed $(p<0.05)$. No significance was showed between the months and groups. Significance between stations and group $(p<0.01)$ and station and month showed significant $5 \%$ level $(p<0.05)$. Based on the benthic abundance/biomass of groups (Three-way ANOVA studies), it further clarified that three areas, namely southern seagrass, northern seagrass and mangroves were significantly different from each other. Bray-

Table 6. Correlation analysis shows relationship between environmental parameters and faunal distribution

\begin{tabular}{|c|c|c|c|c|c|c|c|c|c|c|c|c|c|c|c|c|}
\hline & Clay & DO & Dominance & Evenness & Nitrate & Nitrite & $\mathrm{OM}$ & $\mathrm{pH}$ & Phosphate & Rich & Salinity & Sand & Shanon & Silicate & Silt & Temp \\
\hline Clay & 1 & 0.27 & $-0.67^{*}$ & -0.66 & -0.55 & -0.56 & $0.93^{* *}$ & -0.38 & -0.51 & -0.37 & $-0.88^{* *}$ & $-0.99^{* *}$ & -0.28 & -0.4 & 0.32 & $-0.71^{*}$ \\
\hline DO & 0.27 & 1 & 0.03 & 0.41 & -0.16 & -0.64 & 0.17 & $-0.89 * *$ & 0.6 & 0.49 & -0.25 & -0.34 & $0.74^{*}$ & -0.21 & 0.59 & -0.39 \\
\hline Dominance & $-0.67^{*}$ & 0.03 & 1 & $0.84^{* *}$ & 0.44 & 0.17 & -0.62 & -0.05 & 0.45 & 0.2 & $0.84^{* *}$ & 0.6 & 0.33 & 0.04 & 0.22 & 0.11 \\
\hline Evenness & -0.66 & 0.41 & $0.84^{* *}$ & 1 & 0.3 & -0.08 & -0.65 & -0.26 & $0.75^{*}$ & 0.51 & 0.69 & 0.59 & 0.63 & -0.02 & 0.18 & 0.12 \\
\hline Nitrate & -0.55 & -0.16 & 0.44 & 0.3 & 1 & 0.42 & $-0.72^{*}$ & -0.05 & 0.21 & 0.05 & 0.7 & 0.52 & 0.18 & 0.4 & 0.02 & 0.7 \\
\hline Nitrite & -0.56 & -0.64 & 0.17 & -0.08 & 0.42 & 1 & -0.46 & 0.65 & 0.12 & 0.11 & 0.57 & 0.6 & -0.07 & 0.51 & -0.46 & 0.53 \\
\hline $\mathrm{OM}$ & $0.93^{* *}$ & 0.17 & -0.62 & -0.65 & $-0.72^{*}$ & -0.46 & 1 & -0.23 & -0.51 & -0.22 & -0.86 & $-0.92^{* *}$ & -0.24 & -0.26 & 0.33 & $-0.73^{*}$ \\
\hline $\mathrm{pH}$ & -0.38 & $-0.89^{* *}$ & -0.05 & -0.26 & -0.05 & 0.65 & -0.23 & 1 & -0.36 & -0.22 & 0.27 & 0.48 & -0.57 & 0.12 & $-0.82^{* *}$ & 0.36 \\
\hline Phosphate & -0.51 & 0.6 & 0.45 & $0.75^{*}$ & 0.21 & 0.12 & -0.51 & -0.36 & 1 & $0.74^{*}$ & 0.45 & 0.46 & $0.85^{* *}$ & 0.16 & 0.13 & 0.12 \\
\hline Rich & -0.37 & 0.49 & 0.2 & 0.51 & 0.05 & 0.11 & -0.22 & -0.22 & $0.74^{*}$ & 1 & 0.27 & 0.33 & $0.9^{* *}$ & 0.44 & 0.17 & 0.2 \\
\hline Salinity & $-0.88^{* *}$ & -0.25 & $0.84^{* *}$ & $0.69^{*}$ & $0.7^{*}$ & 0.57 & $-0.86^{* *}$ & 0.27 & 0.45 & 0.27 & 1 & $0.85^{* *}$ & 0.27 & 0.28 & -0.17 & 0.51 \\
\hline Sand & $-0.99^{* *}$ & -0.34 & 0.6 & 0.59 & 0.52 & 0.6 & $-0.92^{* *}$ & 0.48 & 0.46 & 0.33 & $0.85^{*}$ & 1 & 0.2 & 0.35 & -0.45 & $0.72^{*}$ \\
\hline Shanon & -0.28 & $0.74^{*}$ & 0.33 & 0.63 & 0.18 & -0.07 & -0.24 & -0.57 & $0.85^{*}$ & $0.9^{*}$ & 0.27 & 0.2 & 1 & 0.3 & 0.44 & 0.05 \\
\hline Silicate & -0.4 & -0.21 & 0.04 & -0.02 & 0.4 & 0.51 & -0.26 & 0.12 & 0.16 & 0.44 & 0.28 & 0.35 & 0.3 & 1 & 0.17 & 0.66 \\
\hline Silt & 0.32 & 0.59 & 0.22 & 0.18 & 0.02 & -0.46 & 0.33 & $-0.82^{* *}$ & 0.13 & 0.17 & -0.17 & -0.45 & 0.44 & 0.17 & 1 & -0.35 \\
\hline Temp & $-0.71^{*}$ & -0.39 & 0.11 & 0.12 & $0.7^{*}$ & 0.53 & $-0.73^{*}$ & 0.36 & 0.12 & 0.2 & 0.51 & $0.72^{*}$ & 0.05 & 0.66 & -0.35 & 1 \\
\hline
\end{tabular}

Notes: *Correlation is significant at the 0.05 level ( 2 tailed); ${ }^{* *}$ Correlation is significant at the 0.01 level (2-tailed).

Table 7. Three-way ANOVA for comparing between stations, months and benthic groups and first order interaction effects between these three factors

\begin{tabular}{clcc}
\hline Source & \multicolumn{1}{c}{ Sum of squares } & Degree of freedom & \multicolumn{1}{c}{ Mean sum of squares } \\
\hline (A) Stations & 126890.0 & 5 & $(25378.0)$ \\
(B) Groups & 2137510.0 & 8 & $(267189)$ \\
(C) Months & 127698.0 & 23 & 5652.098 \\
A×B & 2989080.0 & 53 & 56397.74 \\
B $\times$ C & 2657300.0 & 215 & 12359.53 \\
A×C & 592078.0 & 143 & 4140.406 \\
AB interaction & & 40 & 18117.0 \\
BC interaction & & 184 & 2130.91 \\
AC interaction & & 115 & 2934.69 \\
Error & 1161150.0 & 920 & 1262.12 \\
Total & & 1295 & $1.681^{* *}$ \\
\hline
\end{tabular}

Notes: * Calculated $F$ is significant at $5 \%$ level $(P<0.05)$; ** Calculated $F$ is significant at $1 \%$ level $(P<0.01)$. 
Curtis similarities were calculated on (root transformed) species dominance data, diversity indices and environmental parameters for the three groups (SSE, NSE and ME) and the resulting dendrogram is shown in Fig. 12. Two distinct benthic community groupings (at $93 \%$ similarity) could be distinguished that apparently reflected differences in sediment/habitat types within Minicoy Island seagrass ecosystem and adjacent mangrove ecosystem. Cluster 1 (PMSSE, PMNSE, PMME, PRSSE, PRNSE, PRME) consisted of three groups (SSE, NSE, ME), outside of the monsoon season, with high diversity, richness and abundance of organisms particularly in the southern seagrass ecosystem (SSE) dominated by Cerithiun spp. and Cardium divarticum assemblage and in northern seagrass ecosystem (NSE) by $\mathrm{Eu}$ rythoe matheii. Cluster 2 (MSSE, MNSE, MME) consisted of all stations during the monsoon season, which is a period characterized by high rainfall and river flow. Cluster 2 consisted of Stas 5 and 6 outside of the monsoon season and was characterized by low species abundance and diversity, along with high organic matter (OM). In MDS plot (Fig. 13), it was found that all the monsoonal samples were ordinated separately from all other samples which confirm the dendogram.

\section{Discussion}

This study gave a reference state of the structural benthic composition of the seagrass and mangrove communities along the Minicoy Island, Lakshadweep. The temperature did not show any marked spatial variations $\left(<1.5^{\circ} \mathrm{C}\right)$ since the stations are close by. During most of the month, the northern seagrass area showed comparatively lower temperature values. The salinity acts a limiting factor in the distribution of living organisms, and its variation caused by dilution and evaporation influences the fauna most likely in the intertidal zone (Gibson, 1982). In the present study, salinity at all the stations was high during pre-monsoon and low during the monsoon season. Higher values in the premonsoon could be attributed to faster evaporation in the study area. Thus, the variation of salinity at study sites is probably due to freshwater runoff and rain. Schrijvers et al. (1998) stated that temperature; salinity and bottom deposits were major factors influencing the distribution of bottom fauna. In the present investigation, dissolved oxygen was high during the monsoon season at all sites, which might be due to the cumulative effect of higher wind velocity coupled with heavy rainfall and the resultant freshwater mixing. Relatively lower values were observed during post-monsoon, which could be mainly due to the reduced agitation and turbulence of the coastal water (Fig. 5). Kundu et al. (2010) have attributed seasonal variations in dissolved oxygen mainly to the freshwater influx and ferriginous impact of sediments. It is well known that the temperature and salinity affect the dissolution of oxygen (Satheeshkumar and Khan, 2012a). Hydrogen ion concentration $(\mathrm{pH})$ in surface waters remained alkaline at all sites throughout the study period, with the maximum value during the premonsoon season and the minimum during monsoon. Generally, fluctuations in $\mathrm{pH}$ values during different seasons of the year are attributed to factors like removal of $\mathrm{CO}_{2}$ by photo-

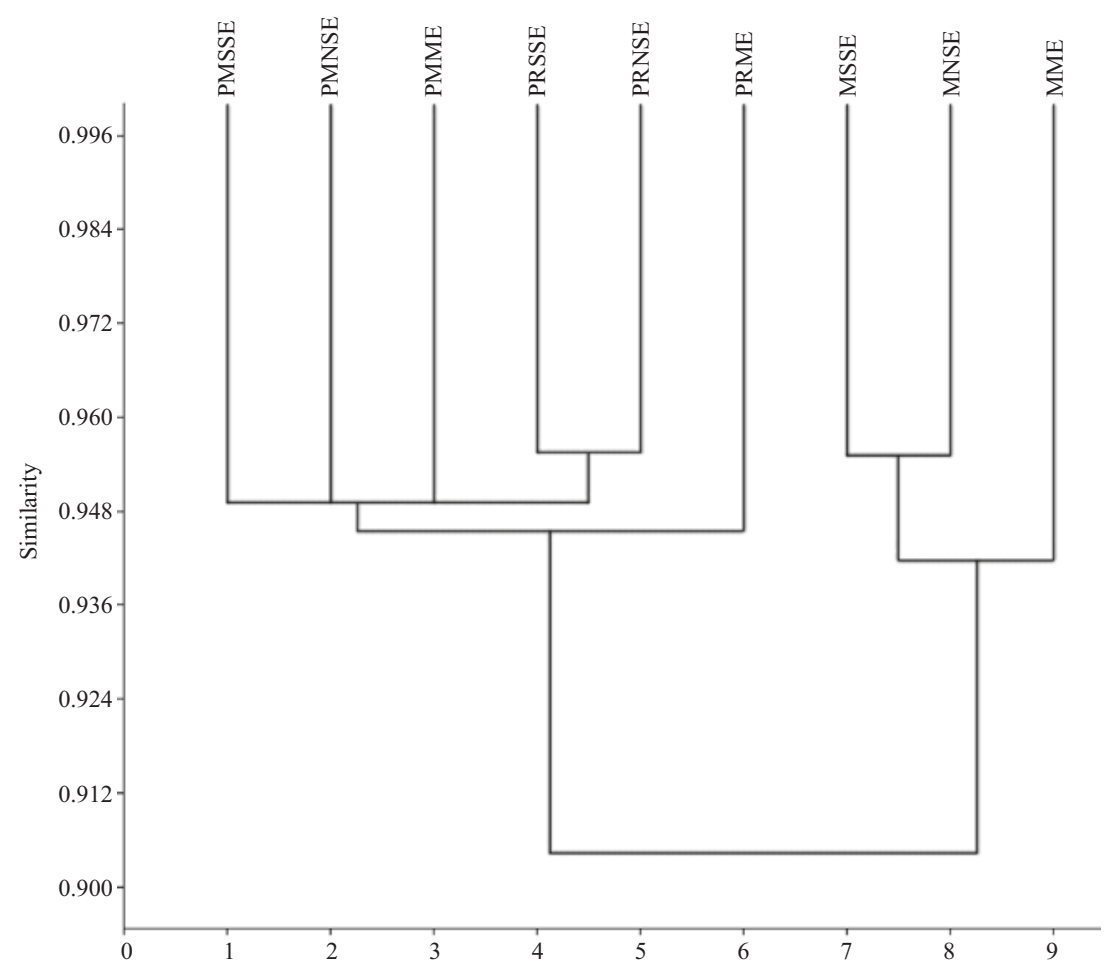

Fig.12. Bray-Curtis similarity dendrogram showing grouping of stations sampled during different. PMSSE represents postmonsoon southern seagrass ecosystem, PMNSE postmonsoon northern seagrass ecosystem, PMME postmonsoon mangrove ecosystem, PRSSE premonsoon southern seagrass ecosystem, PRNSE premonsoon northern seagrass ecosystem, PRME premonsoon mangrove ecosystem, MSSE monsoon southern seagrass ecosystem, MNSE monsoon northern seagrass ecosystem, and MME monsoon mangrove ecosystem. 


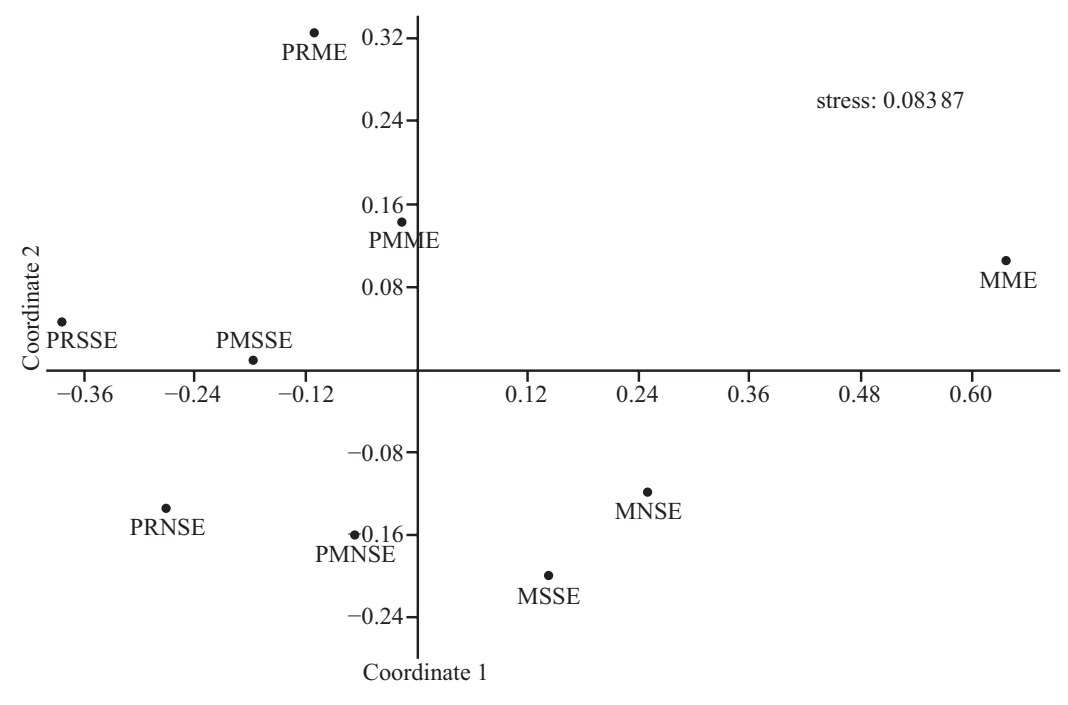

Fig.13. MDS plot similarity showing grouping of stations sampled during different seasons.

synthesis through bicorbonate degradation, dilution of seawater by freshwater influx, reduction of salinity and temperature, and decomposition of organic matter (Kundu et al., 2010; Satheeshkumar and Khan, 2012b).

The present finding that high benthic biomass and density in the southern seagrass region (Thalassia sp. bed) decreased towards the mangrove region and the least was observed in northern seagrass regions, agree with earlier reports from Indian waters (Ansari, 1984; Ansari et al., 1990, 1991). The highest value of biomass was found at Sta. $1\left(181.44 \mathrm{~g} / \mathrm{m}^{2}\right)$ and least was observed at Sta. $4\left(56.98 \mathrm{~g} / \mathrm{m}^{2}\right)$. Highest biomass up to $201.11 \mathrm{~g} /$ $\mathrm{m}^{2}$ of benthic fauna was observed by Ansari et al. (1990) from the coral reef area of Minicoy Island. Similar results are also available from the other parts of the world such as off the Mediterranean basin (Lopez et al., 2010), Inhaca Island of Mozambique (Paula et al., 2001).

Increased dominance in the mangrove sites is due to the high organic carbon, abundance of gastropod species of L. undulata. This showed the ability of these species to survive in adverse conditions, which was similar to the observation of Saravanakumar et al. (2007) from the arid zone of mangroves of the Gulf of Kutch, India. The high organic content of fine fraction most likely determined a reduction in oxygen concentration within the sediments as a result of bacterial and chemical degradation activities (Semprucci et al., 2010). A total of eight major groups identified in the present study were gastropods consisting of 51 species, bivalves of 12 species, soft molluscs (7), polychaetes (27), other worm (7), crabs of 24 species, other crustaceans (including prawns, amphipods, isopods, stomatopods, tanaeids, etc.) of 19 species, echinoderm (11) and sponges of two species leading to a grand total of 160 species from the study area, In the earlier studies from the same area observed that polychaetes were numerically most abundant comprising $60 \%-92 \%$ of total macofauna density and were dominated by suspension and deposit feeding forms (Ansari, 1984). In the present investigation the ostracods and anthozoan groups found in earlier studies were absent and instead echinoderms, sponges, tanaeids, etc. were present.

The present study has shown that sediment characteristics is one of the prime factors controlling the distribution of benthic organisms as most of the benthic group especially polychaetes, molluscs, crabs and amphipods preferred sand dominated sediment texture. Polychaete Eurythoe mathaii were abundant in the fine sand sediment in the present study. Hoey et al. (2004) recorded abundance of Nephtys cirrosa from the similar sedimentary environment in the Belgium Shelf. Furthermore, the occurrences of the bivalve Tellina palatum in large numbers in the sandy sediments of southern seagrass region suggest that this species is a typical inhabitant of this substratum. Species of genus Tellina were also reported from similar sedimentary environments of southern California (Bergen et al., 2000) and North Sea (Duineveld et al., 1991). The abundance of benthic groups especially polychaetes, molluscs and amphipods in southern seagrass region indicated that prevalence of suitable sediment texture for macrobenthic fauna. Presence of isopods, stomatopods in the southern seagrass region may also help in the abundance of other benthic groups.

Species diversity is a simple and useful measure of a biological system. Sanders (1968) found a high level of agreement between species diversity and the nature of the environment and, hence, regarded the measure of species diversity as an ecologically powerful tool. Moreover, Pearson and Rosenberg (1978) proposed that the use of diversity indices is advantageous for the description of faunas at different stages in the succession. Sanders (1968) postulated that the species diversity is mainly controlled by the fluctuations in the environment that lead to less diversity. Species diversity in the present study registered a wide fluctuation between 1.12 (monsoon) at the mangrove site and 3.64 (post-monsoon) at southern segrass region between stations and seasons. The pattern of lower species diversity during monsoon and higher diversity values in post-monsoon recorded in the study area is in conformity with the earlier observations made in Parangipettai coast (Kundu et al., 2010), Godavari mangroves (Raut et al., 2005) and Pondicherry mangroves (Kumar and Khan, 2013). In the present study, richnesses of benthic macro-fauna were the maximum during the postmonsoon season (26.17). A similar observation was reported by Saravanakumar et al. (2007) in Gulf of Kutch mangroves and 
Raut et al. (2005) in Godavari mangroves. The low richness recorded in this study during monsoon (3.21) might be due to the freshwater inflow which induced low saline conditions, which in turn affected the distribution of benthos, particularly, the Polychaetes and molluscs. Maximum diversity and richness recorded during pre-monsoon at the study sites might be due to stable environmental factors, such as salinity, which play an important role in faunal distribution.

CA and MDS have been widely used in the evaluation of spatial and temporal variations in water quality and benthic characteristics of aquatic ecosystems (Satheeshkumar, 2012; Satheeshkumar and Khan, 2012a). Cluster analysis has delineated two major clusters, which were mainly segregated based on seagrass ecosystem and mangrove ecosystem. Clustered stations in the southern seagrass region were densely populated than its northern seagrass region and mangrove sites. The increased abundance, diversity and richness of organisms observed in the southern seagrass region (Stas 1 and 2) could be due to the increased surface area and increased habitat complexity in seagrass regions and abundance of food from decaying seagrass and organic sedimentary material support these large diverse populations of benthic invertebrates in such ecosystem (Ansari, 1984). This could also be due to high energy conditions that keep the organic matter in suspension food for filter feeders. In this case least temporal variations at Sta. $5(42.87 \%)$ and maximum at Sta. $1(79.92 \%)$ were observed. Generally, species richness and density increased with increasing plant biomass at the collection site (Orth, 1973; Ansari, 1984). The results of the present study corroborate these findings. Lower diversity in mangroves may also be attributed to the negative effects of polyphenolic acids derived from mangrove roots, bark and detrital matter, low water content and generally low concentrations of interstitial oxygen and surface micro algae (Schrijvers, 1998). CA was used in this study to examine the differences between the monitoring stations during the four seasons of the year. Based on the cluster analysis, the concentration of dissolved oxygen, $\mathrm{pH}$, salinity and organic matter in the southern seagrass region (Stas 1 and 2) compared high to Stas 3-6. Low DO, high OM and high clay content appear to indicate deteriorating water quality from the standpoint of benthic macroinvertebrate fauna. Human activities have a strong influence on the aquatic environment in the Minicoy Island.

The MDS ordination based on the macrofaunal community clustered the sites into two groups representing three different regions. Group 1 was restricted to the mangrove region, where the Littorina undulata and Scylla serrata predominated within the macrofaunal community. Group 2 comprised SSE and NSR stations of the seagrass region, where opportunistic species such as Cerithium sp., Cardium divarticum, C. asiaticum and Pinna maricata were dominant. These mollusc species were most abundant in seagrass regions, where they DO concentration was high. Among the crustaceans, Isopoda and Amphipoda are also particularly abundant and diverse in the seagrass region, where they are among the most typical members of the benthic communities (Sanders, 1968). Based on the results of multidimensional scale plot suggests that for mangrove benthic macroinvertebrate fauna that environmental parameters such as low DO, salinity, OM and clay have a strong influence on species composition and diversity. From a different multivariate statistical analysis of the different environmental parameters regarding species diversity and abundance of benthic macro- invertebrate fauna, it was found that benthic communities are highly affected by all the environmental parameters governing the distribution and diversity variation of the macrofaunal community in seasgrass and mangrove ecosystem in Minicoy Island, Lakshadweep, India.

\section{Conclusions}

The mangrove zone showed significantly different environmental parameters from the seagrass areas. At the mangrove area lower salinity and DO, higher $\mathrm{pH}$, clay fraction and organic carbon content were noticed than in seagrass areas. In the present study very high species diversity was noticed in southern seagrass stations and northern seagrass stations, but in the mangrove stations comparatively low species diversity was observed. A high biomass was observed in the mangrove stations when compared with their less numerical abundance, due to the large sized gastropod flesh of Terebralia palustris. The temporal distribution of benthos exhibited not only higher density during the post monsoon season but also consisted of more diverse fauna. The decrease of benthic production, especially Polychaetes, during monsoon could be due to the lower temperature and salinity. A medium amount of organic carbon and nutrients phosphorus supports more diverse benthic production. Cluster analysis and multidimensional scale plot analysis were useful helping to define spatial and temporal patterns in seagrass and mangrove ecosystem water quality and macrobenthic fauna characteristics in Minicoy Island. Temperature, dissolved oxygen, salinity, organic carbon, nutrients and sediment characteristics are proved to be important descriptive parameters in terms of the abundance and distribution of benthic organisms. This base line study at Minicoy Island, thus establishes that the benthos of seagrass and mangrove ecosystems (nursery grounds) determines the richness and diversity of demersal fish fauna at the nearby lagoon and reef areas to a great extent. Any serious stress on these ecosystems may further lead to the total disappearance of certain fish species in the nearby future.

\section{Acknowledgements}

The authors are grateful to the Director of Central Marine Fisheries Research Institute, Kochi, India for providing laboratory facilities. The authors thank to reviewers for his invaluable comments to improve the quality of this manuscript.

\section{References}

Alongi D M. 1990. The ecology of tropical soft bottom benthic ecosystems. Ocean Mar Bio Ann Rev, 28: 381-496

Ansari Z A. 1984. Benthic macro and meio fauna of Seagrass (Thalassia hemprichii) bed at Minicoy, Lakshadweep. Ind J Mar Sci, 13(3): $126-127$

Ansari Z A, Ramani P, Rivonker C U, et al. 1990. Macro and meio faunal abundance in six sandy beaches of Lakshadweep Islands. Ind J Mar Sci, 19: 159-164

Ansari Z A, Rivonker C U, Ramani P, et al. 1991. Seagrass habitat complexity and macroinvertebrate abundance in Lakshadweep coral reef regions, Arabian Sea. Coral Reefs, 10(3): 127-131

Bergen M, Weisberg S B, Smith R W, et al. 2000. Relationship between depth, sediment, latitude, and the structure of benthic infaunal assemblages on the mainland shelf of southern California. Mar Biol, 138(3): 637-647

Costanza R, d'Arge R, deGroot R, et al. 1997. The value of the world's ecosystem services and natural capital. Nature, 387(6630): 253- 
260

Day J H. 1967. A monograph on the polychaeta of southern Africa, Part I, Errantia. London: Trustees of the British Museum (Natural History), 1-600

Duineveld G C A, Kunitzer A, Niermann U, et al. 1991. The macrobenthos of the North Sea. Neth J Sea Res, 28(1-2): 53-65

Eleftheriou A, Holme N A. 1984. Macrofauna techniques. In: Holme N A, McIntyre A D, eds. Methods for the Study of Marine Benthos, IBP Handbook 16. Oxford UK: Blackwell Scientific Publications, $140-216$

El-Wakeel S K, Riley J P. 1957. Determination of organic carbon in the marine muds. Journal du Conseil Permanent International Pour Exploration de la mer, 22(2): 180-183

Fernando A, Olivia J F S, 2002. A Field Guide to the Common Invertebrates of the East Coast of India. Annamalai: Annamalai Univerisity, 1-258

Gibson R N. 1982. Recent studies on the biology of intertidal fishes. Ocean Mar Bio Ann Rev, 20: 363-414

Gleason H A. 1992. On the relation between species and area. Ecology, 3(2): 158-162

Heck J R K L, Hays G, Orth R J. 2003. Critical evaluation of the nursery role hypothesis for seagrass meadows. Mar Eco Pro Ser, 253: 123-136

Hemminga M A, Duarte C M. 2000. Seagrass Ecology. Cambridge: Cambridge University Press, 1-298

Hoey G V, Degraer S, Vincx M. 2004. Macro-benthic community structure of the soft bottom sediments at the Belgium continental shelf. Estuar, Coast Shelf Sci, 59(4): 599-613

Khan S A, Raffi S M, Lyla P S. 2005. Brachyuran crab diversity in natural Pitchavaram and artificially developed mangroves Vellar estuary. Current Science, 88: 1316-1324

Krumbein W C, Petttijohn F J. 1983. Manual of Sedimentary Petrography. New York: Appleton Century-Crafts Inc, 549

Kumar P S, Khan A B. 2013. The distribution and diversity of benthic macroinvertebrate fauna in Pondicherry Mangroves, India. Aquatic Bios, 9: 15

Kundu S, Mondal N, Lyla P S, et al. 2010. Biodiversity and seasona variation of macro-benthic infaunal community in the inshore waters of Parangipettai Coast. Enviro Moni Asse, 163(1-4): 67-79

Lopez Y, Royo G, Casazza C, et al. 2010. A biotic index using the seagrass Posidonis oceanic (Bipo), to evaluate the ecological status of coastal waters. Ecol Indi, 10: 380-389

Mullin J B, Riley J P. 1955. The spectrophotometric determination of nitrate in natural water with particular reference to Sea water. Ana Chim Acta, 12: 464-480

Murphy J, Riley J P. 1962. A modified single solution method for the determination of phosphate in natural waters. Ana Chim Acta, 27: $31-36$

Orth R J. 1973. Benthic infauna of eelgrass, Zostera marina, beds. Chesapeake Sci, 14(4): 258-269
Paula J, Fidalgo Ecosta P, Martins A, et al. 2001. Patterns of Abundance of Seagrasses and Associated Infaunal Communities at Inhaca Island, Mozambique. Estuar, Coast Shelf Sci, 53(3): 307-318

Pearson T H, Rosenberg R. 1978. Macrobenthos secession in relation to organic enrichment and pollution of the marine environment. Ocean Mar Bio Ann Rev, 16: 229-234

Pielou E C. 1966. The measurement of diversity in different types of biological collection. Theor Biol, 13: 131-144

Raut D, Ganesh T, Murty N V V S, et al. 2005. Macrobenthos of Kakinada Bay in the Godavari delta, east coast of India: Comparing decadal changes. Estuar, Coast Shelf Sci, 62(4): 609-620

Sanders H L. 1968. Marine benthic diversity: A comparative study. Ame Natur, 102(925): 243-282

Saravanakumar A, Sesh Serebiah J, Thivakaran G A, et al. 2007. Benthic macrofaunal assemblage in the arid zone mangroves of Gulf of Kachchh-Gujarat. J Ocean Uni China, 6(3): 303-309

Satheeshkumar P. 2012. Mangrove vegetation and community structure of brachyuran crabs as ecological indicators of Pondicherry coast, South east coast of India. Iranian J Fish, 11(1): 184-203

Satheeshkumar P, Khan A B. 2012a. Identification of mangrove water quality by multivariate statistical analysis methods in Pondicherry coast, India. Envir Moni Ass, 184(6): 3761-3774

Satheeshkumar P, Khan A B. 2012b. Influence of environmental parameters on the distribution and diversity of molluscan composition in Pondicherry mangroves, Southeast coast of India. Ocean Sci J, 47(1): 61-71

Schrijvers J, Camargo M G, Pratiwi R, et al. 1998. The infaunal macrobenthos under East African Ceriops tagal mangroves impacted by epibenthos. Journal of Experimental Marine Biology and Ecology, 222(1-2): 175-193

Semprucci F, Paola B, Anita M, et al. 2010. Benthic communities along a littoral of the Central Adriatic Sea (Italy). Helg Mar Res, 64(2): 101-115

Shannon C E, Weaver W. 1963. The Mathematical Theory of Communication. Urbana, Illinois: University of Illinois Press, 117

Snedecor G W, Cochran W G. 1967. Statistical Methods. 6th ed. Ames: The Iowa State University Press, 593

Snelgrove P V R. 1998. The biodiversity of macrofaunal organisms in marine sediments. Bio Cons, 7(9): 1123-1132

Strickland J D H, Parsons T R. 1968. A Practical Handbook of Seawater Analysis. Ottawa: Fisheries Research Board of Canada, 167, 311

Strickland J D H, Parsons T R. 1972. A Practical Handbook of Seawater Analysis. 2nd ed. Ottawa: Fisheries Research Board of Canada, Bulletin No 167, 419

Williams A B. 1984. Shrimps, Lobsters and Crabs of the Atlantic Coast Eastern United Stated, Maine to Florida. Washington: Smithsonian Institution Press, 550

Williams S L, Heck K L Jr. 2001. Seagrass community ecology. In: Bertness M D, Gainess S D, Hay M E, eds. Marine Community Ecology. Sunderland: Sinauer, 317-337 